\title{
Incoherent Scatter Radar Studies of Daytime Plasma Lines
}

\author{
Frank T. Djuth ${ }^{1}$ D $\cdot$ Herbert C. Carlson ${ }^{2} \cdot$ Liwei D. Zhang $^{1}$
}

Received: 18 April 2017 / Accepted: 20 February 2018 / Published online: 13 March 2018

(C) The Author(s) 2018

\begin{abstract}
First results from wideband (electron phase energies of 5-51 eV), high-resolution $(0.1 \mathrm{eV})$ spectral measurements of photoelectron-enhanced plasma lines made with the $430 \mathrm{MHz}$ radar at Arecibo Observatory are presented. In the $F$ region, photoelectrons produced by solar EUV line emissions (He II and Mg IX) give rise to plasma line spectral peaks/valleys. These and other structures occur within an enhancement zone extending from electron phase energies of 14-27 eV in both the bottomside and topside ionosphere. However, photoelectron-thermal electron Coulomb energy losses can lead to a broadened spectral structure with no resolved peaks in the topside ionosphere. The plasma line energy spectra obtained in the enhancement zone exhibit a unique relation in that phase energy is dependent on pitch angle; this relation does not exist in any other part of the energy spectrum. Moreover, large fluctuations in the difference frequency between the upshifted and downshifted plasma lines are evident in the 14-27 eV energy interval. At high phase energies near $51 \mathrm{eV}$ the absolute intensities of photoelectron-excited Langmuir waves are much larger than those predicted by existing theory. The new measurements call for a revision/ improvement of plasma line theory in several key areas.
\end{abstract}

Keywords Arecibo radar · Daytime plasma lines · Plasma line energy spectrum · Difference frequency $\cdot$ Plasma line pitch angle dependence

\section{Introduction}

In the past, Langmuir waves (LWs) measured with incoherent scatter radars at $f_{o} \pm f_{r}$, where $f_{o}$ is the radar transmission frequency and $f_{r}$ is the Langmuir wave frequency in the ionosphere (e.g. Evans 1969), have yielded a great deal of information about the ionosphere

Frank T. Djuth

fdjuth@geospace-research.com

1 Geospace Research, Inc., El Segundo, CA, USA

2 Utah State University, Logan, UT, USA 
and thermosphere. The radar signals at $f_{o} \pm f_{r}$ are referred to as plasma lines, PLs, and the upshifted and downshifted signals at offsets of $\pm f_{r}$ are designated as PL+ and PL-, respectively. Usually PL enhancements well above the nighttime thermal level (e.g., Salpeter 1961) are necessary for application oriented studies. Enhancements can be generated by photoelectrons, auroral/magnetospheric particle precipitation, in situ chemical releases/ electron beams, or high-power high-frequency wave-plasma interactions.

The focus of the current work is on high-resolution, photoelectron-enhanced PL (PEPL) spectral features obtained during the most recent solar sunspot maximum (cycle 24) at Arecibo Observatory, Puerto Rico (AO). High resolution techniques developed in the past to study PEPLs include the cutoff technique of Showen (1979), the chirp technique of Hagfors et al. (1984), the application of the coded long-pulse (CLP) technique of Sulzer (1986a) to PEPLs (Djuth et al. 1994) (CLPPL), and high resolution measurements of photoelectron enhanced plasma lines using the alternating code technique (32 bits code) with the EISCAT VHF radar (Guio and Kofman 1996). Such techniques have been used to monitor gravity waves (Vidal-Madjar 1978; Djuth et al. 1994, 1997, 2004, 2010; Livneh et al. 2007, 2009; Nicolls et al. 2014), to develop strategies leading to the assessment of ion composition in the lower thermosphere (Bjørnå and Kirkwood 1988; Fredriksen 1990; Nicolls et al. 2006; and Aponte et al. 2007), and to investigate PL asymmetries at low altitudes (Oran et al. 1978) and the impact of collisions between electrons and neutrals on the PL (Newman and Oran 1981; Bjørnå 1989). The splitting of the PL near the second harmonic of the electron cyclotron frequency has been examined by Bhatt et al. (2008). Fredriksen and La Hoz (1992) used a chirped technique to significantly improve measurements of daytime photoelectrons and field-aligned currents at EISCAT. Also, Fredriksen et al. (1989) demonstrated that electron temperature could be determined with simultaneous EISCAT observations at VHF and UHF, and Fredriksen et al. (1992) performed a detailed study of PEPL intensity versus angle relative to the geomagnetic field $\mathbf{B}$. The PEPL phase energy spectrum has been interpreted within the context of the photoelectron spectrum and several key experimental/theoretical issues have been examined (Cicerone 1974; Oran et al. 1978, 1981; Kofman and Lejeune 1980; Bjørnå and Kirkwood 1986; Guio and Lilensten 1999). Similar measurements have also been used to determine PEPL energy loss and transport versus PEPL energy (Cicerone et al. 1973; Abreu and Carlson 1977). Conjugate photoelectrons were studied at Arecibo by Wickwar (1972). Carlson et al. (1977) included PEPLs in an electron thermal balance study and concluded that the resolution of the factor of two difference between the solar EUV flux and the electron heat balance calculations should be sought in more accurate effective heating rates rather than by simply increasing the EUV flux.

In the current work, studies of the difference frequency $\Delta f_{r}=\left(f_{r+}-f_{r-}\right)$, where $f_{r+}$ and $f_{r \text { - }}$ are the frequencies of the upshifted and downshifted PEPLs, respectively, are included with particular emphasis on PEPL altitude regions containing spectral structure $(14-27 \mathrm{eV})$. There is a long history of experiment and theory in the use of the difference frequency $\Delta f_{r}$ to determine electron temperature and other ionospheric parameters. Measurements of electron temperature and the electron drift velocity in the $F$ region with the $\Delta f_{r}$ technique were pioneered by Showen (1979). In these early experiments the so-called cutoff technique was used, which yielded only one PL+ and one PL- data point at the peak of the $F$ region per profile. Prior to the measurements of Showen (1979), Bauer et al. (1976) suggested the use of the difference frequency to measure field-aligned currents. Electron temperatures were measured with a multistatic version of this technique by Kofman et al. (1981). Hagfors and Lehtinen (1981) describe an electron temperature measuring technique based on simultaneous observations of plasma lines at different spatial scales. 
Behnke and Ganguly (1986) used the cutoff technique along with vertical ion velocities to measure electron currents in the ionosphere above Arecibo. Kirkwood and Bjørnå (1992) performed tristatic PL experiments with EISCAT and obtained accurate measurements of electron temperature in the $F$ region. Heinselman and Vickrey (1992a) showed that the cut-off technique could be used to accurately measure the altitude of the $F$ region peak and small $1 \%$ perturbations in electron concentration near the peak. Heinselman and Vickrey (1992b) indicated that photoelectrons and secondary auroral electrons have a significant impact on the plasma dispersion function and must be accounted for when interpreting $\Delta f_{r}$. This theoretical study was performed in the absence of the geomagnetic field. The impact of heat flow on $\Delta f_{r}$ is discussed by Kofman et al. (1993) and Mishin and Hagfors (1994). Guio et al. (1998) derived the plasma dispersion function and the reduced electron velocity distribution function for an arbitrary velocity distribution with cylindrical geometry along the geomagnetic field as part of an effort to calculate $\Delta f_{r}$. Modeled distributions of photoelectrons and precipitating electrons were included in the calculations. The suprathermal distributions were derived from the angular energy flux calculated by the multistream electron transport model along the Earth magnetic field described in Lilensten et al. (1989). Guio et al. (1998) conclude that the suprathermal electrons have a significant impact on the Langmuir root of the plasma dispersion relation for EISCAT observations made with the VHF (224 MHz) and ESR (500 MHz) radars. On average, the suprathermal electron tail reduces $\Delta f_{r}$ compared to the result obtained for a Maxwellian electron distribution. Subsequently, Guio and Lilensten (1999) examined the effect of the suprathermal electrons on the intensity and Doppler frequency of PLs. Experimental data were acquired by applying the cutoff technique to the EISCAT VHF $(224 \mathrm{MHz})$ radar. The radar was pointed $12^{\circ}$ relative to $\mathbf{B}$. They conclude that a Spitzer function used for the ambient electrons together with a modeled suprathermal distribution function fits the temporal history of PL amplitude and Doppler data the best. Nicolls et al. (2006) used the Arecibo line feed pointed in the vertical direction and the CLPPL technique to obtain $\Delta f_{r}$ versus altitude in the ionospheric environment near solar minimum (i.e. 2006). Electron temperatures in the upper thermosphere $(>200 \mathrm{~km})$ were convincingly measured with this technique.

This paper is organized in the following manner. In Sect. 2.1 the observed wideband (5-51 eV) PEPL spectrum is discussed, and its compatibility with existing theory is examined. In Sect. 2.2 special attention is focused on the fact that the phase energies of the observed PEPL spectral structures between 14 and $27 \mathrm{eV}$ have a pitch angle dependence. In Sect. 2.3 it is shown that the spectral structures have a significant impact on $\Delta f_{r}$. The observations are discussed within the context of existing geomagnetic models and plasma theory in Sect. 3, and Sect. 4 summarizes the principal conclusions of the present work.

\section{Observations}

The Arecibo $430 \mathrm{MHz}$ radar is sensitive to only those Langmuir waves that have the same magnitude and direction as the effective two-way radar wavevector $\boldsymbol{k}$. Also, the Langmuir wave phase velocity $v_{\phi}$ is defined as $2 \pi f_{r} / k$ and the Langmuir wave phase energy is $E_{\phi}=1 / 2 m_{e} v_{\phi}^{2}$, where $f_{r}$ is the Langmuir wave frequency, $k \cong 18 \mathrm{~m}^{-1}=4 \pi / \lambda$ (Bragg condition, where $\lambda$ is the wavelength of the radar transmissions), and $m_{e}$ is electron mass. The Langmuir wave frequency $\left(\omega_{r} \equiv 2 \pi f_{r}\right)$ can be expressed to second order for $k_{ \pm} \lambda_{D} \ll 1$ as

$$
\omega_{\mathrm{r} \pm}^{2}=\omega_{\mathrm{pe}}^{2}+\sin ^{2} \theta \omega_{\mathrm{ce}}^{2}+\frac{3 \mathrm{k}_{\mathrm{r} \pm}^{2} \kappa T_{\mathrm{e}}}{m_{\mathrm{e}}},
$$


where $\omega_{\mathrm{pe}}=\left(n_{e} e^{2} / \varepsilon_{\mathrm{o}} m_{e}\right)^{1 / 2}$ and $\omega_{\mathrm{ce}}$ are the electron plasma frequency and the electron cyclotron frequency, respectively; $n_{e}$ is electron concentration, $e$ is electron charge, $\varepsilon_{\mathrm{o}}$ is the permittivity of free space, $m_{e}$ is electron mass, $\theta$ is the angle between the radar line-of-sight and the geomagnetic field $\mathbf{B} ; k_{ \pm}=2 \pi / c\left[f_{o}+\left(f_{o} \pm f_{r}\right)\right]$ is radar wavenumber corrected for wave propagation downward (+, upshifted Doppler) and upward (-, downshifted Doppler) (e.g., Showen 1979); $f_{o}$ is the radar operating frequency; $\kappa$ is Boltzmann's constant; $T_{e}$ is electron temperature; $\lambda_{D}$ is the Debye length $\left(\varepsilon_{0} \kappa T_{e} / n_{e} e^{2}\right)^{1 / 2}$; and $c$ is the speed of light. The frequency offsets from $430 \mathrm{MHz}$ of the signal peaks at PL+ and PL- are the same as $f_{r+}$ and $f_{r-}$, respectively.

Physically, the thermal ionospheric plasma gives rise to Langmuir waves (day and night) as part of the electron Landau damping process. Locally produced photoelectrons (PEs) and conjugate PEs streaming through the ionosphere generate Langmuir waves via a resonant electron Landau damping process wherein the velocity of the PE is slightly greater than the phase velocity of the excited Langmuir wave (e.g., Perkins and Salpeter 1965). The PE Langmuir wave amplitudes are generally much greater than their thermal counterpart. The corresponding PE intensity expressed in absolute $\kappa T_{\mathrm{p}}\left(E_{\varnothing}\right)$ units is $25-160$ times greater than the nighttime thermal level depending on $f_{r}$ and the level of EUV emissions. In the theory of Yngvesson and Perkins (1968-hereafter $Y P$ ) absolute Langmuir wave energy $\kappa T_{\mathrm{p}}\left(E_{\varnothing}\right)$ is expressed as:

$$
\kappa T_{p}=\kappa T_{e} \frac{f_{m}\left(E_{\phi}\right)+f_{p}\left(E_{\phi}\right)+\chi}{f_{m}\left(E_{\phi}\right)-\kappa T_{e} \frac{d f_{p}\left(E_{\phi}\right)}{d E_{\phi}}+\chi},
$$

where $f_{\mathrm{p}}$ is the one-dimensional velocity distribution of the PEs measured at the radar wave vector and expressed as $E_{\phi} ; f_{\mathrm{m}}$ is a modified one-dimensional velocity distribution of the ambient electrons, which includes the effects of increased electron Landau damping resulting from the Arecibo radar line-of-sight relative to $\mathbf{B}$; and $\chi$ provides for excitation and damping of plasma waves by the collective effects of electron-ion collisions. The quantities $f_{\mathrm{m}}$ and $\chi$ can be readily calculated from the measured values of $T_{\mathrm{e}}, n_{e}$, the geomagnetic field strength $|\mathbf{B}|$, the angle between the radar wave vector and $\mathbf{B}$, and radar wavelength.

At very low $E_{\varnothing}$ in (2), one has $\kappa T_{\mathrm{p}} \sim \kappa T_{e}$, that is, a strong thermal $\left(f_{\mathrm{m}}\right)$ Langmuir wave excitation term exists. For example if foF2 is $\sim 4.2 \mathrm{MHz}$ during the daytime, and $T_{e}$ is $2500 \mathrm{~K}$, the Langmuir waves are primarily excited thermally (e.g., Salpeter 1961). As $E_{\varnothing}$ increases, $f_{\mathrm{p}}$ becomes dominant and $\kappa T_{\mathrm{p}}$ rises sharply. At somewhat greater $E_{\phi}$, the photoelectron Landau damping term $-\kappa T_{e}\left(\mathrm{~d} f_{p} / \mathrm{d} E_{\phi}\right)$ dominates the Langmuir wave damping provided that large photoelectron fluxes are present. At very large $E_{\phi}, \chi$ dominates both the Langmuir wave excitation and damping processes.

The initial PL work at Arecibo Observatory (AO) focused on daytime PEPLs and addressed issues related to the PE energy spectrum $(Y P)$ and electron thermal balance in the midlatitude $F$ region (e.g. Carlson et al. 1977). In this work and in the present study $430 \mathrm{MHz}$ radar measurements of thermal electron temperatures $T_{\mathrm{e}}$, electron density $n_{e}$, and PEPLs are made. Absolute photoelectron-enhanced Langmuir wave intensities $\left(\kappa T_{p}\right)$ versus electron phase energy $E_{\phi}$ are of interest, where $T_{p}$ is defined as the apparent, velocity-dependent, plasma temperature of a hypothetical, random, non-interacting electron gas necessary to produce the Langmuir wave intensities measured with the Arecibo radar (Perkins and Salpeter 1965; YP; Fremouw et al. 1969). In the past there was a 24-eV $E_{\phi}$ limitation set by the frequency response of the $430 \mathrm{MHz}$ line feed. The AO Gregorian feed has a wider PL+ bandwidth and was used in the acquisition of the data presented here. 
The primary radar observing program entailed the application of the coded long-pulse (CLP) technique to daytime PLs (Sulzer 1986a; Djuth et al. 1994). This is referred to as the CLPPL technique. A digital receiver (Echotek GC214) with two $5 \mathrm{MHz}$ bandpass channels was used to record the CLPPL data. The Gregorian feed combined with the CLPPL technique offers continuous PL coverage throughout the $F$ region at an electron phase energy resolution of $\sim 0.1 \mathrm{eV}$ and electron phase energies up to $51 \mathrm{eV}$ in the present study. Higher values of $E_{\phi}$ are achieved with greater values of foF2. In addition, with the CLPPL technique the $\kappa T_{p}$ measurement errors are more than an order of magnitude smaller than those encountered in past experiments of this nature.

It is important to note that the $430 \mathrm{MHz}$ radar views only a narrow band of photoelectron energies at one angle relative to $\mathbf{B}$ (i.e. the pitch angle) in each radar range gate. For example, at $f_{r+}=5 \mathrm{MHz}\left(E_{\phi+}=8.537 \mathrm{eV}\right)$ the maximum $E_{\emptyset}$ bandwidth, set by the spectral width of the PL (PL+ or PL-) measured on a steep $F$ region gradient is only $\sim 0.08 \mathrm{eV}$ Full Width at Half Maximum (FWHM), and the nominal value is $\sim 0.03 \mathrm{eV}$ FWHM. However, each range cell contains a broad spectrum of photoelectron energies $(\sim 1$ to $\sim 70 \mathrm{eV})$ spread across a large pitch angle distribution that cannot be monitored with the AO radar. The Bragg condition required for backscatter with the $430 \mathrm{MHz}$ radar, $k \cong 18 \mathrm{~m}^{-1}=4 \pi / \lambda$, together with Eq. (1), which is dominated by the term $\omega_{p e}^{2} \propto n_{e}$, results in a single measurable value of $E_{\varnothing}$ at each range cell. A wideband radar spectrum of the PEPL is obtained by using data from many consecutive radar range cells along the PL altitude profile, which nominally extends from a lower altitude in the range $120-150 \mathrm{~km}$ to a higher altitude between $650 \mathrm{~km}$ and $800 \mathrm{~km}$ depending on ionospheric conditions, time of day, etc. Each range cell yields a $\kappa T_{p}$ value for a given $f_{r}$, or equivalently $E_{\varnothing}$, and by definition this gives rise to two separate $E_{\varnothing}$ spectra, one for the bottomside ionosphere and one for the topside. The PEPL signals are limited by electron Landau damping on the topside and electron Landau damping plus electron-neutral collisions on the bottomside to $f_{r}$ values greater than about 3.3 MHz (i.e., $3.7 \mathrm{eV}$ for PL+ and $3.8 \mathrm{eV}$ for PL-).

\subsection{Wideband PEPL Spectrum}

In the current work PEPL measurements made with the CLPPL technique were augmented with analyzed data from a standard AO incoherent scatter program, Multiple Radar Autocorrelation Function (MRACF) (Sulzer 1986b). MRACF was run serially with CLPPL to provide background electron and ion temperature and ion velocity data. During the observations reported here radar time was shared between two projects involving the same investigator group. The projects consisted of the current research study and one dealing with electron thermal balance in the ionosphere. This latter required a strategic reduction of the radar range resolution from $150 \mathrm{~m}$ (best possible) to $300 \mathrm{~m}$. However, this did not impact the accuracy of the $\kappa T_{p}\left(E_{\phi}\right)$ values in the current investigation. Radar echoes from PEPLs appear as narrow spectral peaks centered near $430 \mathrm{MHz}+f_{r}$ and $430 \mathrm{MHz}-f_{r}$; the two peaks correspond to Langmuir wave vectors directed toward and away from the radar, respectively. The Gregorian feed exhibits good performance up to the largest available zenith angle $\left(19.6^{\circ}\right)$. PL+ is used in broadband experiments because of the greater Gregorian feed bandwidth available above $430 \mathrm{MHz}(\sim 13 \mathrm{MHz})$ compared to the bandwidth below $430 \mathrm{MHz}(\sim 8 \mathrm{MHz})$.

The PL $\kappa T_{\mathrm{p}}\left(E_{\varnothing}\right)$ spectrum is obtained by measuring $f_{r}$ (the exact frequency location of the PL peak) versus altitude as well as the power in the PL peak (i.e., the integrated area under the plasma line peak) versus altitude. As part of a preprocessing procedure, the data are evaluated for external interference and the presence of artifacts. Large amplitude, 
narrow band (less than $\sim 10 \mathrm{kHz}$ ) interference is automatically removed from the data, and artifacts and wideband interference are either eliminated with special purpose software or the affected data are flagged and removed from the dataset. The PL peak location, its amplitude, its width, and a baseline offset are determined via a nonlinear least-squares fitting procedure. An overview of the implementation of this technique is provided in Djuth et al. (1994). The integrated area is expressed in terms of absolute Langmuir wave energy $\kappa T_{\mathrm{p}}$ with the aid of the radar system constant developed for the Gregorian feed. The signals in the radar range profile of $\kappa T_{\mathrm{p}}$ are then mapped to $f_{r}$ with the aid of the $f_{r}$ range profile, and then to $E_{\phi}=1 / 2 m_{e}\left(2 \pi f_{r} / k_{+}\right)^{2}$. Figure 1 shows an example of the PL+ (i.e., $\left.f_{r+}\right)$ profile obtained on 12 April 2012. The Gregorian beam was pointed at $15^{\circ}$ zenith angle and $168^{\circ}$ in azimuth, that is, in the direction of geomagnetic South at an angle relative to $\mathbf{B}$ that ranged from $30.95^{\circ}$ at $120 \mathrm{~km}$ altitude to $32.02^{\circ}$ at $650 \mathrm{~km}$ altitude. Because $n_{e}$ is roughly proportional to $f_{r}^{2}$, the profile indicates that a steep vertical $n_{e}$ gradient was present in the bottomside $F$ region.

The unique aspect of the April observations is that they were made near the vernal equinox at solar maximum when the peak plasma density in the Arecibo ionosphere is large. In Fig. 1 the PL+ value at the $F$ region peak is $12.14 \mathrm{MHz}$, and the altitude of the peak is $311.1 \mathrm{~km}$. This profile was measured in the middle of the 12 April 2012 observation period extending from 1411:38 to 1501:04 AST. The high PL values near $12 \mathrm{MHz}$ are indicative of a very strong $F$ region ionosphere at AO. This resulted in the highest $f_{r}$ values by far under which $\kappa T_{p}\left(E_{\varnothing}\right)$ has been measured to date, and as a result the electron phase energy

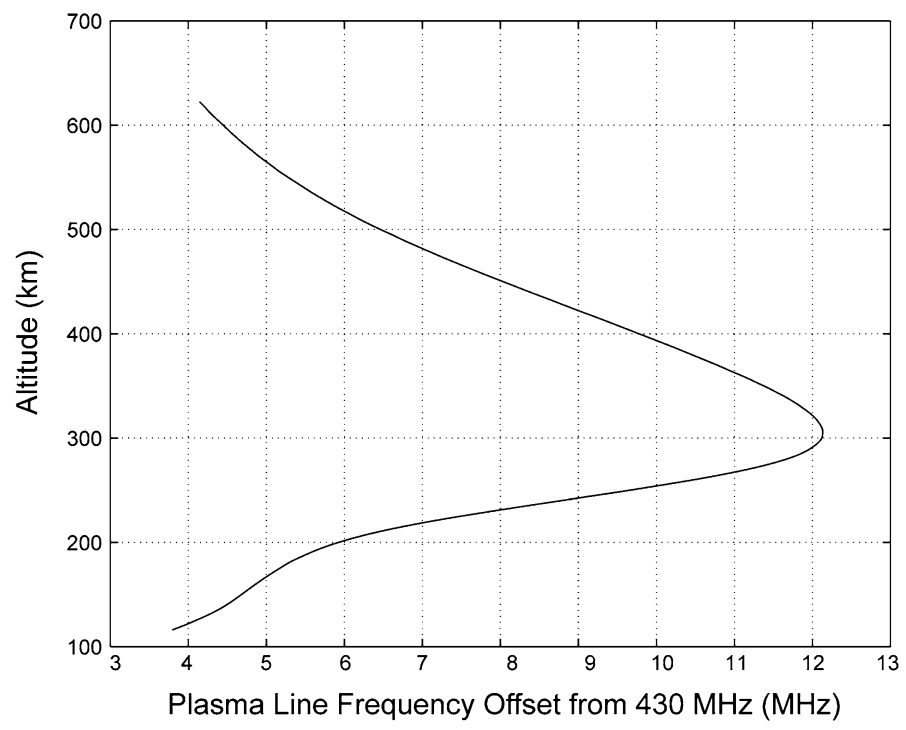

Fig. 1 Upshifted plasma line profile measured between 1435:29 and 1435:39 AST on 12 April 2012. The peak value of the plasma line frequency is $12.14 \mathrm{MHz}$, and the peak altitude is $311.1 \mathrm{~km}$. The \pm one standard deviation error bars $( \pm 1 \sigma)$ for the plotted data points are too small to display. The worst case nonlinear least-squares fitting errors are along the steep vertical gradient near $230 \mathrm{~km} \pm 7 \mathrm{~km}$ where the $\pm 1 \sigma$ errors are approximately $\pm 0.0264 \%$ of the plotted values, and at $\sim 425 \mathrm{~km} \pm 15 \mathrm{~km}$, where the errors are approximately $\pm 0.0221 \%$. The smallest errors are near $311.1 \mathrm{~km}$ (the F region peak), where the errors are $\pm 0.0019 \%$ of the plotted values, and above $\sim 550 \mathrm{~km}$ where the errors are $\pm 0.0012 \%$ [see e.g., Djuth et al. (1994)] 
coverage was very large. In order to acquire the PL+ data throughout the ionosphere, the two AO 5-MHz bandwidth digital receiver channels were combined with $100 \mathrm{kHz}$ overlap to obtain full coverage of the PL from 3.3 to $13.2 \mathrm{MHz}$. The consolidation of the two channels was done with great care because a slight change in gain between the two receiver channels has a significant impact on the $\kappa T_{p}\left(E_{\emptyset}\right)$ spectrum (discussed below). The measured gain correction for the upper band $(8.2 \mathrm{MHz}-13.2 \mathrm{MHz})$ relative to the lower band (3.3-8.3 MHz) was small, $-0.034 \mathrm{~dB}$. In Fig. 2, $f_{r}$ is displayed as a function of time and altitude along with simultaneous measurements of $T_{e}$. The peak $f_{r}$ value increases from 11.91 to $12.40 \mathrm{MHz}$ during the $\sim 50 \mathrm{~min}$ period of observations presented. Values of $f_{r}$ on the bottomside of the profile increase slowly with time, and there is a small amount of downward curvature present in the $f_{r}$ temporal development near $240 \mathrm{~km}$ altitude. On the topside $f_{r}$ exhibits a small secular height increase which is most evident near $430 \mathrm{~km}$ altitude. Overall, this is a very stable ionosphere in which to investigate daytime PEPL spectra.

The electron temperature profile in Fig. $2 \mathrm{~b}$ exhibits a minimum near $370 \mathrm{~km}$ altitude. This is the result of an increase in the electron-ion cooling rate $=\mathrm{C}\left(M_{i}\right) n_{e}\left(T_{e}-T_{o+}\right)$ $\left(n_{o+} / T_{e}^{3 / 2}\right)$, where $\mathrm{C}$ is a constant and $n_{e}=n_{o+}$, where $n_{o+}$ is oxygen ion density (e.g., Lei et al. 2007; Schunk and Nagy 2009). The cooling scales approximately as $f_{r}^{4}$, and $f_{r}$ values near $12 \mathrm{MHz}$ result in a dramatic reduction in $T_{e}$. The $T_{e}$ profile combined with the $f_{r+}$ profile indicates that at high altitudes near $600 \mathrm{~km} f_{r+}$ is small $(\sim 4.1 \mathrm{MHz})$ but $T_{e}$ is large $(\sim 1800 \mathrm{~K})$. As a result, electron Landau damping by thermal electrons gives rise to an intensity damping decrement for Langmuir waves of $\sim 10^{3} \mathrm{~s}^{-1}$ (e.g., $Y P$ ), and electron-ion collisions add $\sim 260 \mathrm{~s}^{-1}$. At lower altitudes near $220 \mathrm{~km}, T_{e}$ is also high but $f_{r+}$ is moderately large $(\sim 7.3 \mathrm{MHz})$. In this case the impact of electron Landau damping on Langmuir wave damping is negligible because of the high $f_{r+}$, and the intensity damping decrement

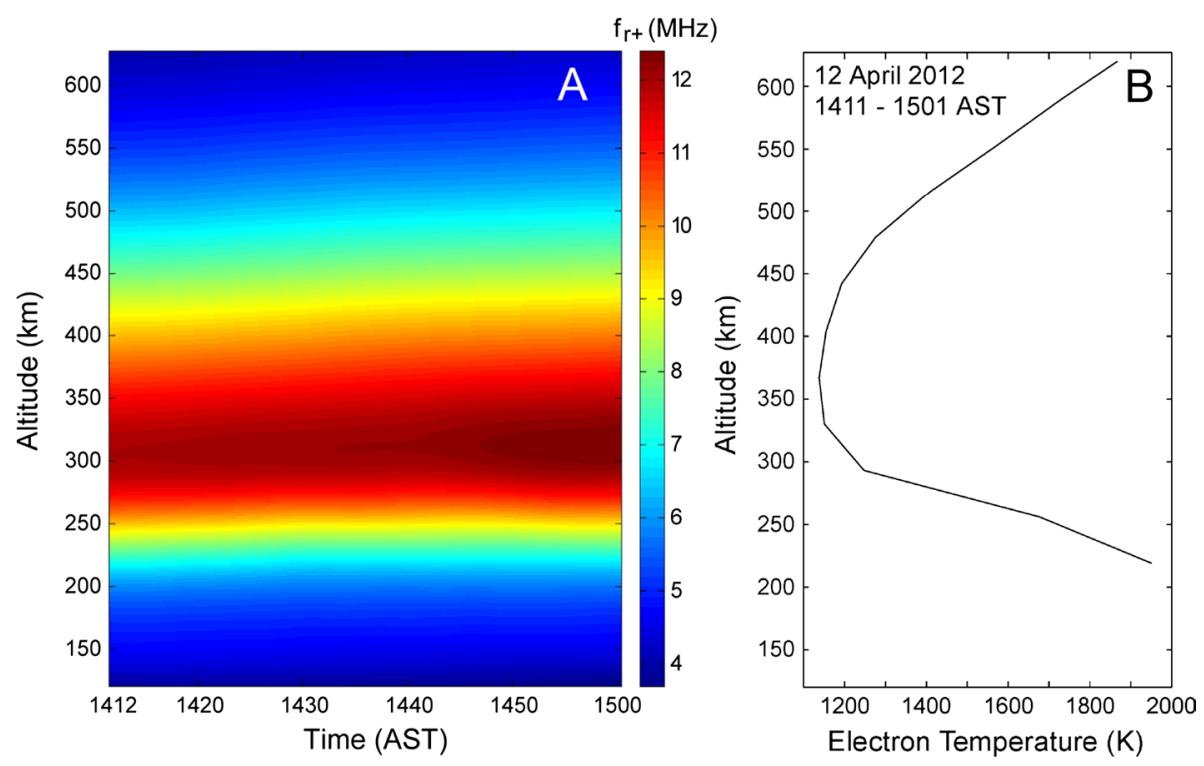

Fig. 2 Upshifted plasma line frequency $f_{r+}$ versus altitude and time on 12 April 2012 (a). Peak values of $f_{r+}$ increase from 11.91 MHz at 1411:38 AST to $12.40 \mathrm{MHz}$ at 1501:04 AST. The electron temperature profile averaged over this period is shown on the right (b). The $\pm 1 \sigma$ error bars for the $T_{e}$ measurements are $\pm 0.2 \%$ of the plotted value at all altitudes displayed 
from electron-ion collisions is $\sim 400 \mathrm{~s}^{-1}$. At middle heights near $310 \mathrm{~km}$, the Langmuir wave damping is also controlled solely by electron-ion collisions. However because of the large $f_{r+}$ value $(\sim 12 \mathrm{MHz})$ and low $T_{e}$ value $(\sim 1200 \mathrm{~K})$ this region generates a large intensity damping decrement of $\sim 2700 \mathrm{~s}^{-1}$. In Sect. 3, this damping rate is found to be important in determining whether PLs observed at high $E_{\varnothing}(\sim 51 \mathrm{eV})$ are fully excited by PEs.

The values of $f_{r+}$ versus height and time in Fig. 2 a can be converted ito $E_{\varnothing}$ versus height and time to facilitate the mapping of $\kappa T_{p}$ versus altitude and time to $\kappa T_{p}$ versus $E_{\varnothing}$ and time. Side-by-side displays of the two $\kappa T_{p}$ plots are presented in Fig. 3. The data in Fig. 3 are grainy because the signal is stochastic. A cubic least-squares 11-point smoothing filter (Savitzky and Golay 1964) has been applied to the original raw $\kappa T_{p}\left(E_{\phi}\right)$ data in the vertical direction for presentation purposes. The data in Fig. 3a are binned in $0.1 \mathrm{eV}$ increments. A gap near $51 \mathrm{eV}$ is evident in Fig. 3a because initially the peak $f_{r+}$ value $(11.91 \mathrm{MHz}$ ) was not large enough to support a maximum electron phase energy of $51 \mathrm{eV}$ (i.e. $f_{r+}=12.32 \mathrm{MHz}$ ). The gap gradually fills in with time, but the net result is greater $\kappa T_{p}$ error bars in Fig. 5 (discussed below) between 47 and $51 \mathrm{eV}$. The $\kappa T_{p}$ bottomside structures evident in Fig. 3a between phase energies of 15.2 and $25.2 \mathrm{eV}$ map to altitudes between $\sim 224$ and $\sim 246 \mathrm{~km}$ in Fig. $3 \mathrm{~b}$. Notice that the $\kappa T_{p}$ temporal variation at altitudes below $250 \mathrm{~km}$ in Fig. $3 \mathrm{~b}$ exhibits the same slight curvature as the observations in Fig. 2. This arises because of the relationship between electron phase energy $\left(\alpha f_{r}^{2}\right), \kappa T_{p}$, and altitude. The nominal height of the $F$ region peak in Fig. $3 \mathrm{~b}$ is $317 \mathrm{~km}$, and it is characterized by a minimum in $\kappa T_{p}$. Values of $\kappa T_{p}$ are smallest at this altitude because $E_{\emptyset}$ is the greatest, and as a result photoelectron fluxes further down in the tail of the distribution function excite the PL. Structure in the topside is more difficult to see in Fig. 3a. The raw voltage signal is weaker than on the bottomside, but $\kappa T_{p}$ values are about the same owing mostly to the
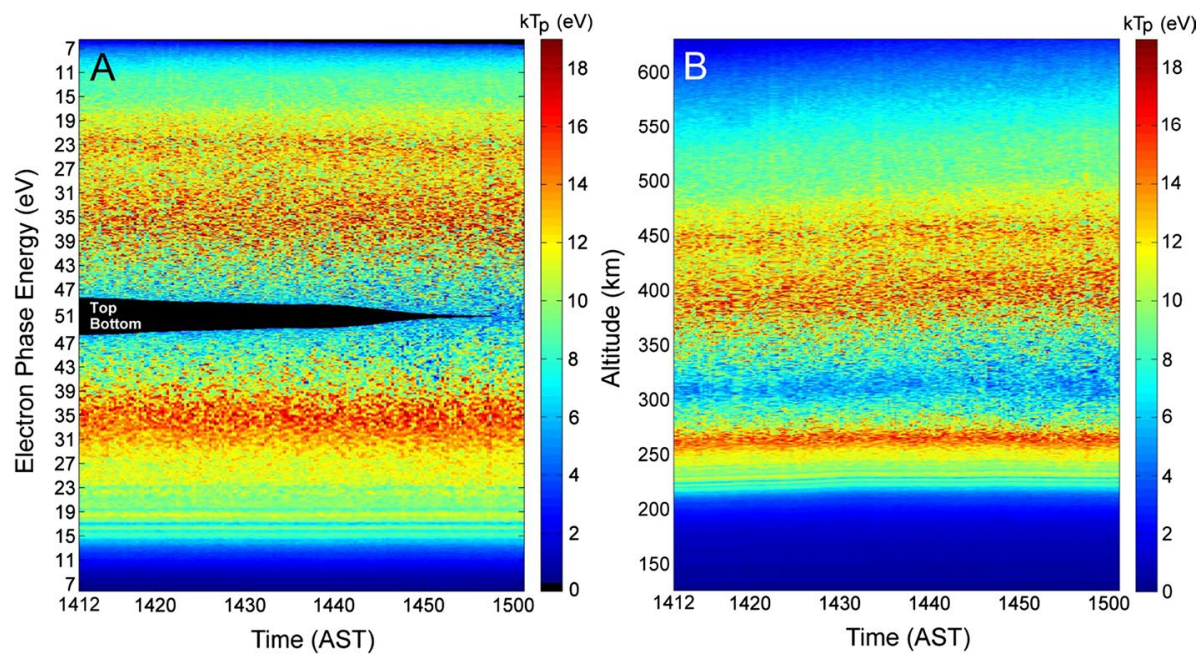

Fig. 3 Side-by-side displays of $\kappa T p$ versus time and electron phase energy obtained using the results of Fig. 2 (a), and $\kappa T p$ versus altitude and time (b) measured on 12 April 2012. The exact time interval for each plot is 1411:38-1501:04 AST and 20-s temporal integrations of the data were employed. Results from the ionosphere from 125 to $630 \mathrm{~km}$ are shown. Layers/structures evident in (b) between $\sim 223$ and $\sim 250 \mathrm{~km}$ altitude map to phase energies between 15.0 and $27.0 \mathrm{eV}$ in (a). A more detailed view of this region is provided in Fig. 4. Also evident are two enhanced topside regions centered near $400 \mathrm{~km}$ and $450 \mathrm{~km}$ in (b) that map to phase energies of $31-39 \mathrm{eV}$ and $18-26 \mathrm{eV}$ in (a) 
range-squared correction that must be applied to the data. Nevertheless, a peak in the PL backscatter is readily seen to be near $E_{\varnothing}=35 \mathrm{eV}$, which is similar to that evident on the bottomside.

To better illustrate the mapping between altitude and $E_{\varnothing}$ we have expanded the observations in Fig. 3a between electron phase energies of 14.2 and $25.3 \mathrm{eV}$ and mapped the observed spectral lines to altitudinal $\kappa T_{p}$ peaks and valleys present in Fig. $3 b$. These results are presented in Fig. 4. In this case we used the raw data with no smoothing. The data in Fig. $4 \mathrm{a}$ is a bit grainier that those of Fig. $4 \mathrm{~b}$ because the size of the $E_{\varnothing}$ pixel is $0.1 \mathrm{eV}$ in the vertical direction, whereas the altitudinal pixel has a $300 \mathrm{~m}$ height in Fig. 4b. The latter generates pixels that are four times smaller in the vertical direction.

A single $\kappa T_{p}\left(E_{\varnothing}\right)$ spectrum can be obtained by integrating the original PL+ raw data underlying the display in Fig. 3a across the 50 min time interval 1411:38-1501:04 AST. This result is presented in Fig. 5. Note that $\kappa T_{p}\left(E_{\varnothing}\right)$ is not the PE flux, and that a PL spectrum of this nature cannot readily be converted to the 3D photoelectron velocity distribution function of $(Y P)$, which requires a smoothly varying PL spectrum. $(Y P)$ fit a cubic to their spectral data before solving their inversion Eq. (18). Large noise-like fluctuations in the one-dimensional photoelectron velocity distribution function and the follow-on 3D distribution function $(Y P)$ result when one attempts to solve Eq. (18) with the irregular/ variable data from Fig. 5. The abscissa in Fig. 5 shows three scales of interest to the plotted data: electron phase energy, PL frequency, and PL altitude. In addition the background electron temperatures averaged over the integration period of the plot are shown at the bottom. The $\pm 1 \sigma$ error bars are displayed as black vertical lines with a dot in the center. The error bars displayed in this figure are dependent on the random errors calculated for each $\kappa T_{p}\left(E_{\phi}\right)$ data point as well as the number of points in each $E_{\phi}$ bin. The latter quantity is strongly dependent on $f_{r+}$ and the vertical $n_{e}$ gradient; the width of each bin is fixed at

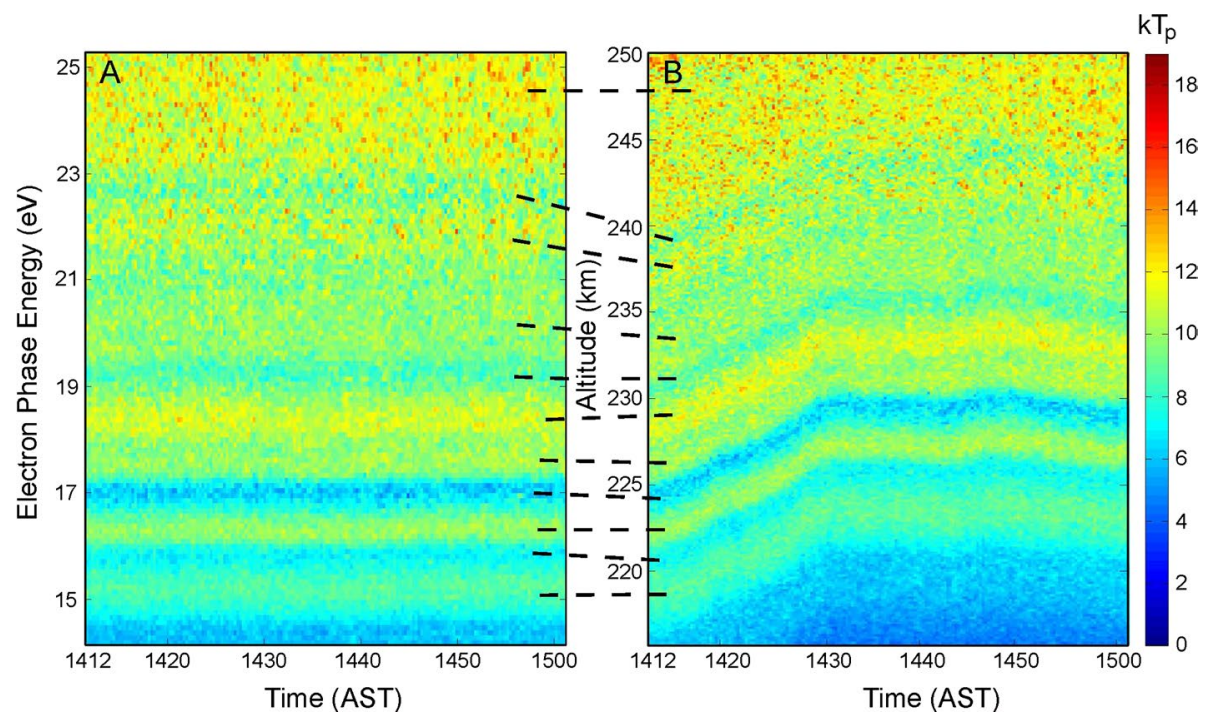

Fig. 4 Magnified view of the electron phase energy in Fig. 3a illustrating the mapping of $\kappa T_{p}$ structure at low altitudes in Fig. $3 b$ to electron phase energy. Dashed lines connect the altitudinal maxima/minima layers in (b) to the corresponding spectral peaks and valleys in (a). Raw unfiltered data were used to construct the above two panels 


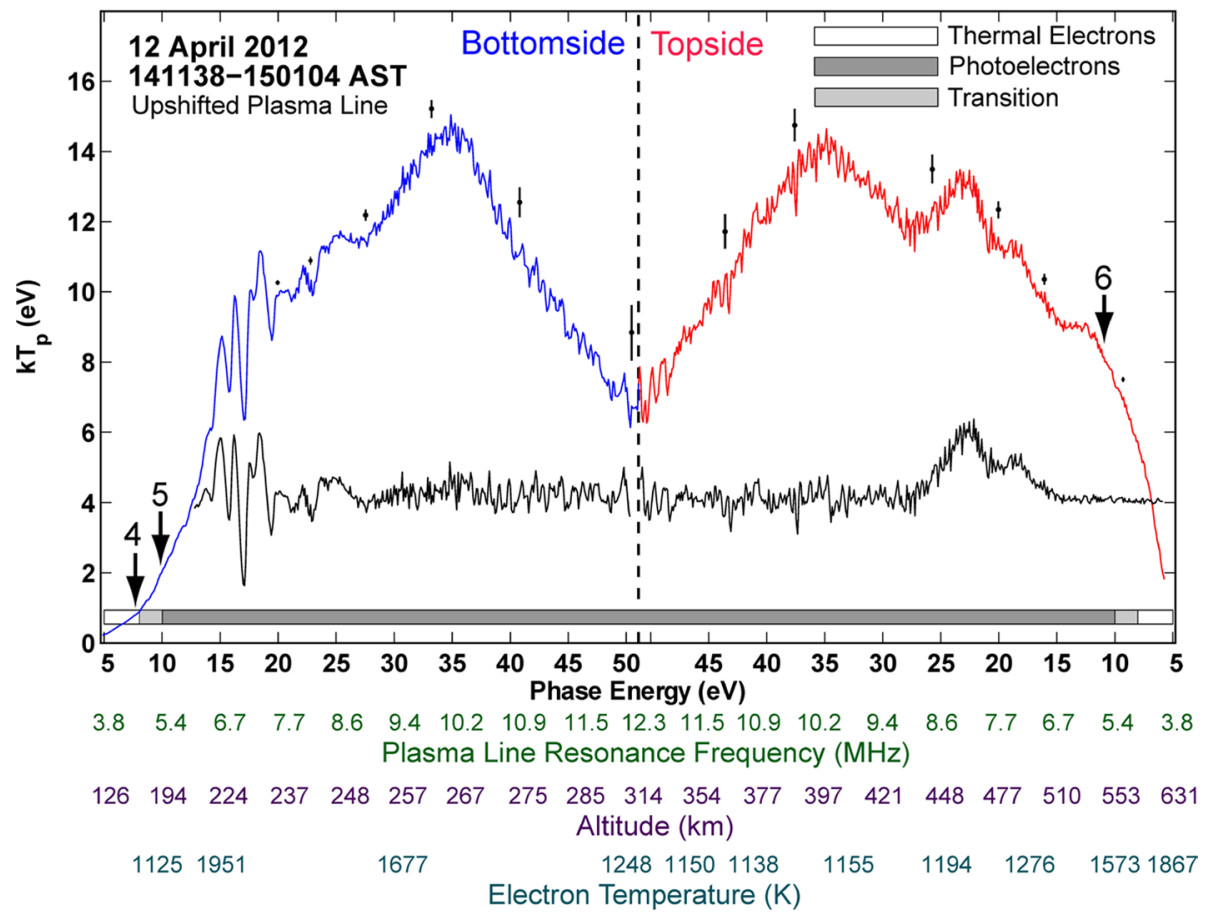

Fig. 5 Plasma line energy (power per unit bandwidth) expressed as the absolute quantity $\kappa T_{p}$ versus electron phase energy for simultaneous measurements made in the bottomside and topside ionosphere. Blue designates data obtained below the $F$ region peak, whereas the red curve indicates data above the $F$ region peak. The black curve is the result of background subtractions from the blue and red curves. See text for details

$0.1 \mathrm{eV}$. A linear five-point smoothing filter has been applied to the region of greater error from $E_{\varnothing}=34.7 \mathrm{eV}$ in the bottomside ionosphere to $28.7 \mathrm{eV}$ in the topside ionosphere. The approximate frequency bands representing Langmuir waves excited by daytime thermal electrons, followed by a transition region, and the spectral region of PE enhancements are shown on a gray scale. Arrows mark the points where $f_{r+}=l \times f_{c e}$, where $l$ is an integer. The rapid fall off of $\kappa T_{p}\left(E_{\varnothing}\right)$ on the topside between 550 and $620 \mathrm{~km}$ altitude at $E_{\varnothing}$ less than $10 \mathrm{eV}$ is the result of the electron Landau damping as discussed in reference to Fig. 2. The spectrum in black shown at the bottom of the plot is the residual spectrum containing spectral peaks/structure after background spectrum subtraction. The baseline is set at $\kappa T_{p}=4 \mathrm{eV}$. Background subtraction involved the use of a FIR filter to remove high frequency spectral peaks/structure. The residual background spectrum was then nonlinear least-squares fit to a polynomial, which was subsequently subtracted from the main spectrum to yield a spectrum containing peaks/structure only. This procedure was performed to remove skewing of spectral peaks/structure by the rising (bottomside) and declining (topside) background spectrum.

On 12 April 2012 the solar sunspot number was 37 and the monthly mean was 55; the F10.7 flux was 95.8 and the daily average of the Kp index was 3-. At the middle of the observation period (1436 AST) the solar zenith angle at AO was $32.5^{\circ}$ and the zenith angle at the conjugate point (near Mar del Plata, Argentina) was $\sim 60.9^{\circ}$. In part, the above parameters are provided to facilitate future modeling of the $\kappa T_{p}\left(E_{\phi}\right)$ spectrum in Fig. 5. 
The first interesting feature of Fig. 5 is that $\kappa T_{p}\left(E_{\varnothing}\right)$ has a substantial component at the electron phase energy of $51 \mathrm{eV}\left(\kappa T_{p}=7.1 \mathrm{eV}\right)$. Prior to this investigation $\kappa T_{p}\left(E_{\varnothing}\right)$ could only be measured to $\sim 24 \mathrm{eV}$ at AO (e.g., Carlson et al. 1977, YP). The observation of a significant $\kappa T_{p}\left(E_{\phi}\right)$ value at $51 \mathrm{eV}$ is in sharp contrast to the predictions of $Y P$, which entail the excitation and damping of Langmuir waves by electron-ion collisions at phase energies near $51 \mathrm{eV}$. If the formalism of $Y P$ were correct one would have $\kappa T_{p}\left(E_{\varnothing}\right) \sim \kappa T_{e}=0.103 \mathrm{eV}$, which is much less than the observed $\kappa T_{p}$ of $7.1 \mathrm{eV}$. The origin of this discrepancy can be traced back to the theory of Perkins and Salpeter (1965), who in their Eq. (46) assume that the high energy portion of the PE tail $\left(E_{\varnothing}>30 \mathrm{eV}\right)$ can be replaced by a Maxwellian. This greatly reduces the intensity in the tail of the PL spectrum determined in their Eq. (47) to $k T_{e}$. Equation (7) of $Y P$ is a reformatted version of Eq. (47) of Perkins and Salpeter (1965). At the time that Perkins and Salpeter (1965) was written the PE production spectrum was thought to fall off sharply after $30 \mathrm{eV}$, whereas we now know that the spectrum extends out to $\sim 70 \mathrm{eV}$. Perkins and Salpeter (1965) and $Y P$ will be corrected as part of a future publication. We note that the calculated PE angular velocity distribution of Guio et al. (1998) does not have the above problem. However, this calculation/formalism is currently only in the $\mathbf{B}$ field-aligned direction.

The second important result of Fig. 5 is the presence of peaks and other structure in the $\kappa T_{p}\left(E_{\emptyset}\right)$ observations. Spectral structure is present in both the bottomside and topside ionosphere. The structure extends over the $E_{\varnothing}$ interval $\sim 14$ to $\sim 27 \mathrm{eV}$ on both the bottomside and the topside. However, the sharpest spectral peaks and valleys are observed on the bottomside. The locations of the all bottomside and topside peaks and valleys at phase energies between 14 and $27 \mathrm{eV}$ follow the empirically derived pitch angle function $E_{\phi}(\theta)=D \cos (\theta)^{1.94}$, where $D$ is a normalization constant, and $\theta$ is the angle between the radar beam and $\mathbf{B}$, which is the same as the pitch angle $\alpha$. This formula is fully justified in Sect. 2.2. As noted above the radar beam was positioned at $15^{\circ}$ zenith angle and pointed South in the geomagnetic meridian plane. The angle between the radar beam and the region of bottomside $\kappa T_{\mathrm{p}}$ structure centered near $225 \mathrm{~km}$ altitude was $31.54^{\circ}$. Values for $\mathbf{B}$ were obtained from the most recent 12 th generation IGRF model. The peaks and valleys in Fig. 5 are scaled to the "non-magnetized" plasma value of $\theta=\alpha=0$ (i.e. propagation parallel to $\mathbf{B}$ ) and listed in column 5 of Table 1 . The locations of the peaks correspond to the well-known energies of PEs ejected from $\mathrm{N}_{2}$ and $\mathrm{O}$ by the strong solar EUV lines of $\mathrm{Mg}$ IX $36.807 \mathrm{~nm}(33.7 \mathrm{eV})$, and He II $30.378 \mathrm{~nm}(40.8 \mathrm{eV})$ and $25.632 \mathrm{~nm}$ (46.4 eV) (Goembel et al. 1997). Column 6 of Table 1 shows that the same PE peaks have been previously seen with the high-resolution PE spectrometer onboard the AE-E satellite (e.g., Doering et al. 1973, 1976; Lee et al. 1978, 1980a, b; Hernandez et al. 1983; and Goembel et al. 1997). Also between 243 and $248 \mathrm{~km}$ altitude the radar detected a broad 31.8-35.2 eV peak that is evident in column 5 and Fig. 5. The peak is broad because it encompasses three AE-E peaks, and at these higher altitudes all three peaks are beginning to widen due to the Coulomb PE-thermal electron energy loss process (hereafter Coulomb) (e.g., Schunk and Hays 1971; Swartz et al. 1971) discussed below. Langmuir wave harmonics of the electron cyclotron frequency are in the thermal plasma line realm/transition zone and are far away from the structure noted here. The well-defined peaks and valleys observed at electron phase energies between 14 and $27 \mathrm{eV}$ are not observed in the topside ionosphere due to broadening/smearing by large Coulomb losses; they are prominent only between 215 and $248 \mathrm{~km}$ altitude where Coulomb losses are small. An identical topside broadening phenomenon was observed with the AE-E PE spectrometer at altitudes above $300 \mathrm{~km}$ (Lee et al. 1980b). 


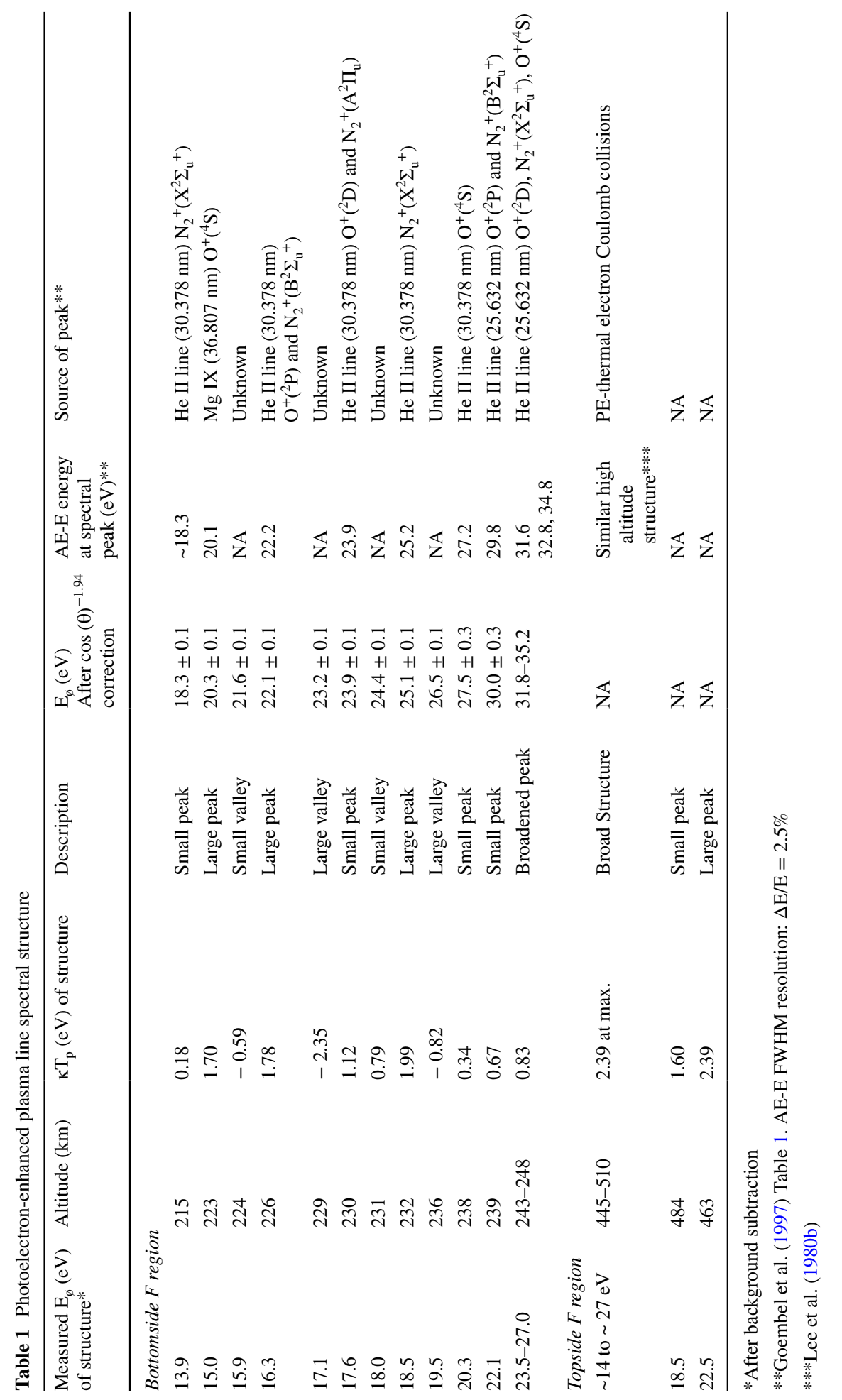


Figure 6 shows where the bottomside and topside peak/valley spectral band resides along the simultaneously measured electron density profile. The electron density at the topside spectral band is lower than the bottomside band because of Coulomb losses, particularly at lower energies (e.g. Swartz et al. 1971). As noted above, the Coulomb loss process is also responsible for peak broadening in the topside spectra. Local Coulomb losses from PE local production (within a few $\mathrm{km}$ of the observing point) are not large enough to completely explain the width/smearing of the topside broadened echo. The downward transport of PE flux to the topside spectral measurement location is required to achieve the observed broadening as well as the enhanced spectral signal strength. Although the PE flux traveling towards the radar observing point from the conjugate hemisphere is degraded upon reaching the $\sim 460$ to $\sim 500 \mathrm{~km}$ altitude interval, the downward flux necessary to augment the observed spectral broadening and spectral amplitude in this region is certainly present. Previously broad PE spectra above $300 \mathrm{~km}$ were measured with the AE-E satellite with a look direction at the dip equator towards the north. These data were interpreted by Lee et al. (1980b) as follows. The measured thermal electron density at the spacecraft $N_{p}$ was assumed to be proportional to the total electron content (TEC) down to the "production altitude" near $300 \mathrm{~km}$, and an exponential increase in electron density from the satellite altitude to $\sim 300 \mathrm{~km}$ altitude was employed based on AE-E results. Good agreement between the smearing of the EUV line-generated spectral peaks and TEC anchored to $N_{p}$ was evident in the observations. Although AE-E measured PEs moving upwards to

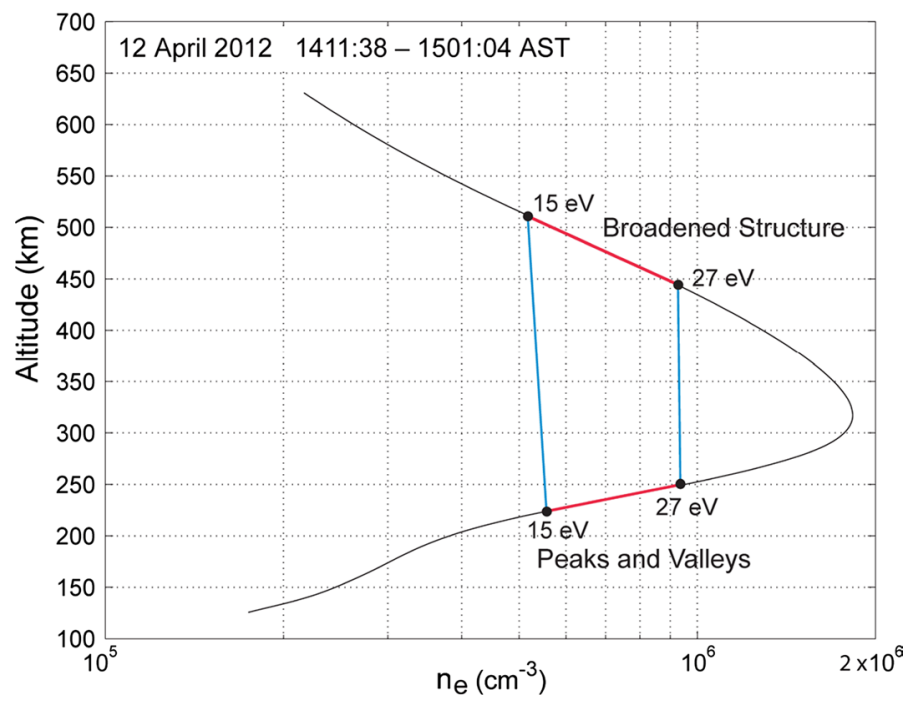

Fig. 6 Electron density $\left(n_{e}\right)$ profile corresponding to the observations of Fig. 5. It was obtained using the frequency of the spectral peak in the upshifted plasma line spectrum versus altitude. This corresponds to the frequency profile of $\omega_{r+}$ shown in Eq. (1). Measured values of electron temperature (Fig. 2) are combined with the IGRF geomagnetic field values to obtain $\omega_{p e}$ from which $n_{e}$ can be calculated. The effect of $T_{e}$ on $n_{e}$ is small (maximum of $1 \%$ for the current profile), and the dependence on $\omega_{c e}$ is negligible. The profile is averaged over the same period as the spectrum in Fig. 5. The region of the $n_{e}$ profile where peaks and valleys occur as a result of solar EUV emissions is shown as a red overtrace on the bottomside $F$ region. A similar topside overtrace marks the broadened spectral structure. The $E_{\emptyset}$ spectral bandwidth is approximately the same in both cases. The topside spectrum spreads to lower $n_{e}$ values than the corresponding bottomside spectrum. Blues lines connect the spectral regions to aid in judging $n_{e}$ differences 
the satellite (while in the Northern Hemisphere), and the data in Fig. 6 is for PEs moving downward toward the radar, the basic concept is that the TEC between the PE observing point and the PE source point(s) determines the level of smearing of the transported PEs. This is further supported by the radar observations of Abreu and Carlson (1977). With the approximation of Mantas et al. (1978) $\mathrm{d} E / \mathrm{d} x=-\beta n_{e}(x) / E$, where $\beta=3 \times 10^{-12}$ $\mathrm{eV}^{2} \mathrm{~cm}^{2}$, and $E$ is PE energy, one finds that at $3 \mathrm{~km}$ (local) distance the lowering of $E$ is only $\sim 0.03 \mathrm{eV}$ at both spectral end points 14 and $27 \mathrm{eV}$ on the topside of Fig. 6. In order to generate the observed width/smearing it takes at least 100 times the TEC used in the above estimate of local losses. This provides additional support for the concept that conjugate PEs are responsible for the observed broadened topside spectra.

\subsection{Pitch Angle Dependence}

Results of experiments performed in February and September 2013 showed signs that the $E_{\varnothing}$ and $v_{\phi}$ at the $\kappa T_{p}$ spectral peaks/valleys were dependent on $\theta$. This effect was experimentally investigated on 19 February 2015 by first scanning the radar in zenith angle and then subsequently scanning in azimuth angle. Figure 7 shows the downshifted plasma line frequency $f_{r \text { - }}$ (i.e. the PL- spectral peak frequency) versus altitude and time during the zenith angle scans from $~ 0703$ AST to 0930 AST. The radar beam was initially used to scan the

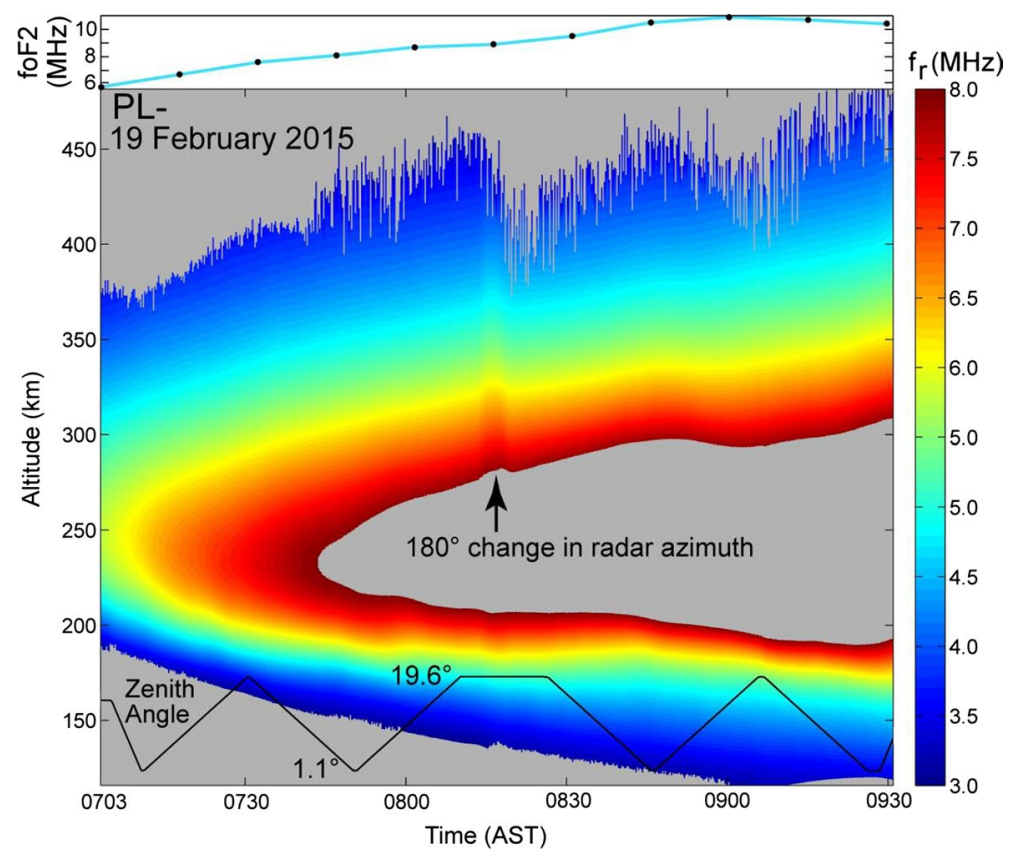

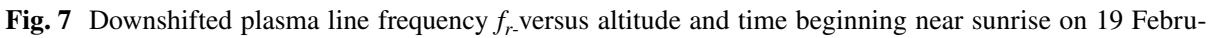
ary 2015. Initially the radar beam was pointed in the geomagnetic South direction. An arrow near 0815 AST marks the time when the radar feed arm was rotated from its initial position in the geomagnetic South meridian plane to the North meridian plane. During the repositioning process the beam moved across the enhanced electron density region east of $\mathrm{AO}$ at sunrise. The corresponding values of foF 2 are displayed at the top. Gray designates the area where the reduced Chi squared fit probability is less than $0.5 \%$. The gray gap in the middle is the result of the $8 \mathrm{MHz}$ cutoff of the radar receiver signal 
maximum Gregorian zenith angle range from $1.1^{\circ}$ to $19.6^{\circ}$ in the geomagnetic meridian plane facing South, that is, over angles that were more closely aligned with $\mathbf{B}$. The azimuth was then rotated $180^{\circ}$ at $\sim 0815$ AST, and scans in the North meridian plane were performed that were more closely perpendicular to B. In Fig. 8, the PE-enhanced plasma line energy (integrated area under the PL- spectral peak at each range cell) is displayed in absolute $\kappa T_{p}$ units versus time and altitude. However no radar gain calibration for a diffuse target currently exists when the viewing direction is between $15^{\circ}$ and $19.6^{\circ}$ in zenith angle. In Fig. 8, the gain value for $15^{\circ}$ was used for this scan interval, and as a result $\kappa T_{p}$ is underestimated in this scan range. On 19 February 2015 the solar sunspot number was 61 and the monthly mean was 45 ; the F10.7 flux was 116.0 and the daily average of the Kp index was 2. At the middle of the observation period (0815 AST) the solar zenith angle at $\mathrm{AO}$ was $71.9^{\circ}$ and the zenith angle at the conjugate point was $56.5^{\circ}$. The South scans are shown in greater detail in Fig. 9. As noted above in reference to the April 2012 observa-

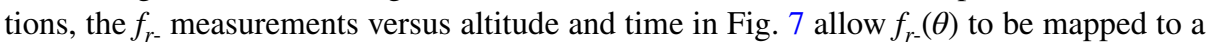
specific altitude and time in Figs. 8 and 9.

Sunrise conditions were selected so that the topside lines above $250 \mathrm{~km}$ would not be excessively broadened by Coulomb losses. At altitudes between 175 and $350 \mathrm{~km}$ the radar beam scan intervals relative to $\mathbf{B}$ (i.e. $\theta$ ) were approximately $27.7^{\circ}$ to $46.2^{\circ}$ in the South and $46.0^{\circ}$ to $64.5^{\circ}$ in the North. Note that the $\kappa T_{p}$ at the spectral peaks is smallest in the South when the zenith angle is $1.1^{\circ}$ where $\theta$ is greatest. The curved $\kappa T_{p}$ structure evident during the South scans is generated by the spectral peaks and valleys shown in Fig. 5 as the

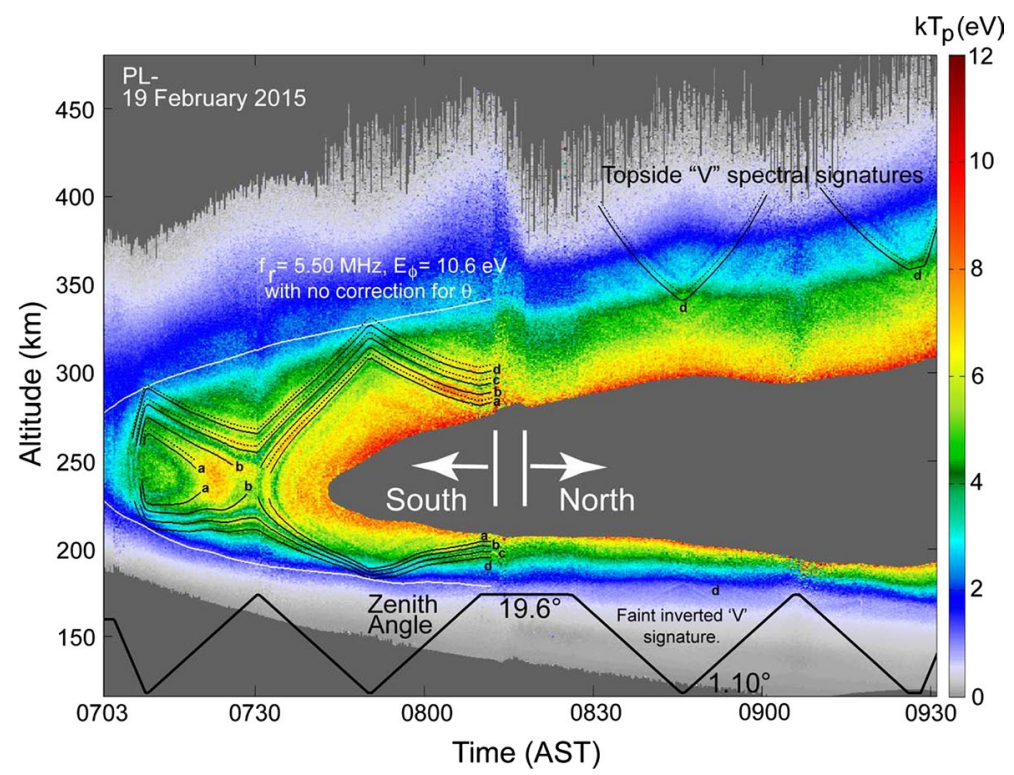

Fig. 8 Radar scans of plasma line $\kappa T_{p}$ versus altitude and time showing the movement of the PE spectral peaks versus zenith angle. Lower case letters are used to identify the PE energy at $\theta=0$ that is responsible for the spectral line (Table 1, column 5). Thus, $a=25.1 \mathrm{eV}, b=23.9 \mathrm{eV}, c=22.1 \mathrm{eV}$, and $d=20.3 \mathrm{eV}$. As in Fig. 7, gray designates the area where the reduced Chi squared fit probability is less than $0.5 \%$. The gray gap in the middle is the result of the PL- signal exceeding the $8 \mathrm{MHz}$ cutoff of the radar receiver, and therefore no signal is present. See text for additional description 


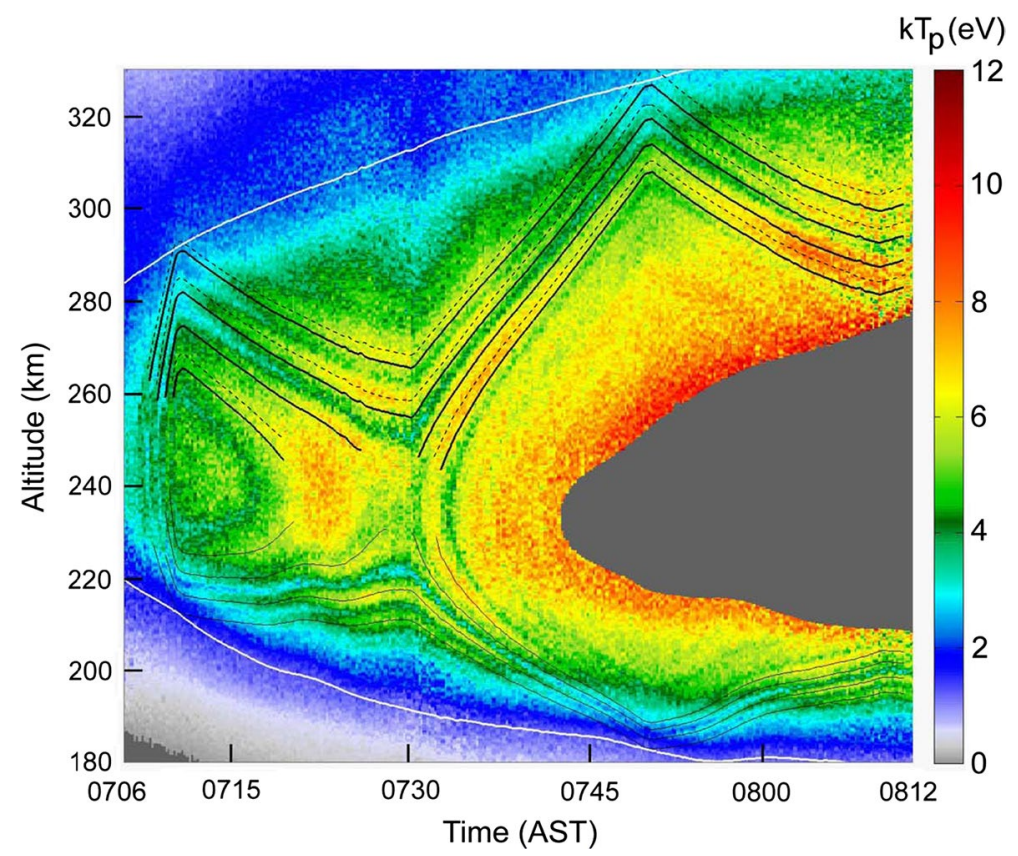

Fig. 9 Temporal expansion of the first hour of data in Fig. 8 showing good detailed agreement between the pitch angle formula in Eq. (3) and the observations below $\sim 240 \mathrm{~km}$ altitude where the photoelectron-thermal electron Coulomb energy loss rate is low

radar beam moves across the morning $F$ region that is increasing in electron density and altitude extent with time. The solid black lines in Figs. 8 and 9 that follow the measured spectral peaks/valleys are calculated using the empirically derived pitch angle formula:

$$
f_{r}(\theta)=A \cos (\theta)^{0.97},
$$

where $A$ is a normalization constant. Thus in the region of spectral peaks/valleys $f_{r}$, and therefore $E_{\varnothing} \propto f_{r}^{2}$, is a function of $\theta$. The letters next to the black curves in Fig. 8 designate the $E_{\phi}(\theta)$ values of the curves at $\theta=0$. This is the unique identifier for a curve that changes energy value as a function of $\theta$. For comparison, a white line in Fig. 8 shows the corresponding curve for $f_{r}=5.5 \mathrm{MHz}(10.6 \mathrm{eV})$, which is outside (lower in energy than) the region of EUV line generated peaks, and therefore the above $\theta$ dependence does not apply. The white curve is the well-known contour for $f_{r}$ and $E_{\varnothing}$ values that are isotropic in $f_{r}$ and $E_{\varnothing}$ and satisfy the standard dispersion relation for Langmuir waves in Eq. (1). The normalization factor $A$ in Fig. 8 is nominally $A=f_{r}\left(31.6^{\circ}\right) /\left(\cos \left(31.6^{\circ}\right)\right)^{0.97}$, where $31.6^{\circ}$ is the $\theta$ at which $\kappa T_{p}$ spectral peaks are observed when the radar is pointed South at $15^{\circ}$ zenith angle during the tests. However, the calculated $\theta$ values take into account changes in the geomagnetic dip angle as a function of altitude, lateral distance, and direction from $\mathrm{AO}$, and as a result $A$ varies with the exact location at which a particular spectral peak is calibrated. The actual normalizing $\theta$ values for Fig. 8 range from $31.59^{\circ}$ to $31.63^{\circ}$. In addition it is important to note that when one compares data from different years the change in the AO dip angle with time must be taken into account. The IGRF model currently includes a secular decrease of $\sim 0.185^{\circ}$ per year for the dip angle above AO. It is of interest to compare the results of the pitch angle formula (3) with data acquired during different years at $\mathrm{AO}$. To do 
this one must take into account the above change in dip angle versus time, which changes the pitch angle of a radar line-of-sight having the same geographic pointing direction in different years. In addition, one must adjust the frequency of the plasma line data used in the two measurements if one measurement is made with $f_{r-}$ and the other is made with $f_{r+}$ since the two frequencies are not exactly the same. This is evident from Eq. (1). With appropriate modifications for the changing $\mathbf{B}$ field versus time and an adjustment for the use of $f_{r-}$ in Fig. 8 instead of $f_{r+}$ in Fig. 5, one can compare the bottomside $f_{r+}$ values of the spectral peaks derived from the $2012 E_{\phi}$ observations in column 1, Table 1 with the bottomside $f_{r \text { - }}$ measurements of Fig. 8 made in February 2015. Several strong peaks at measured $E_{\phi}$ values in column 1 of Table 1 were selected for this study: 15.0, 16.6, 17.6, and $18.5 \mathrm{eV}$. These $E_{\phi+}$ data were converted to $f_{r+}$ values, and the resulting $f_{r+}$ values were offset by $f_{r+}-f_{r-}$ to convert them from $f_{r+}$ to $f_{r-}$. In this case frequency offsets were calculated using Eq. (1), and the offsets were of the order of $1 \mathrm{kHz}$. Comparisons between 2012 and 2015 $f_{r}$ values were then made. In the notation below, the $f_{r d}$ values are derived from the 2012 Table 1 results and corrected for the B-field dip angle of 2015 and for $f_{r \text { - }}$ used in the 2015 tests; $f_{r e}$ are the measured 2015 estimates based on the 20-s averages in Fig. 8. The results are: $15.0 \mathrm{eV}\left(f_{r d}=6.58 \mathrm{MHz}, f_{r e}=6.62 \mathrm{MHz}\right), 16.3 \mathrm{eV}\left(f_{r d}=6.83 \mathrm{MHz}, f_{r e}=6.88 \mathrm{MHz}\right)$, $17.6 \mathrm{eV}\left(f_{r d}=7.11 \mathrm{MHz}, f_{r e}=7.10 \mathrm{MHz}\right)$ and $18.5 \mathrm{eV}\left(f_{r d}=7.28 \mathrm{MHz}, f_{r e}=7.31 \mathrm{MHz}\right)$, where the energy values are the measured values in column 1 of Table 1 . On average $f_{r e}$ exhibits a $\sigma$ of $0.5 \%$ relative to the $f_{r d}$ results. Thus, there is excellent agreement between the predictions of Eq. (3) and the geomagnetic South data in the region below the $F$ region peak ( $233 \mathrm{~km}$ altitude) in Figs. 8 and 9.

Dashed lines are plotted in the topside $F$ region of Figs. 8 and 9 to indicate the location of the spectral peaks, whereas the solid lines show $f_{r-}(\theta)$ for the hypothetical case where there is no energy loss. On the topside there is a slight positive offset between the altitude of the peak $\kappa T_{p}$ and that calculated from the pitch angle function which signifies energy loss (i.e. $f_{r-}$ or $\mathrm{E}_{{ }_{\phi-}}$ decreases with increasing altitude). This occurs primarily because of Coulomb losses. The travel distance of a PE to account for the spreading and displacement of the topside peaks is $\sim 25-50 \mathrm{~km}$ depending on PL spectra location along the $n_{e}$ profile. Figure 10 shows where the measured PL peaks and valleys generated by solar EUV line emissions reside on the $n_{e}$ profile at 07:42 AST (just before loss of signal at the $F$ region peak). Only the strongest spectral peaks labeled "a" through "d" are clearly observed at this time. As noted earlier there is a difference in $n_{e}$ values associated with the EUV PL spectral bands in the topside versus the bottomside $F$ region because of Coulomb losses. In the topside $F$ region Coulomb losses contribute to the PL spread to lower $E_{\phi}$ and therefore to lower $n_{e}$. Other processes such as the forward scattering of the PEs off of ions, O, and $\mathrm{N}_{2}$, and inelastic PE excitation of $\mathrm{O}\left({ }^{1} \mathrm{D}\right)$ leading to a $1.97 \mathrm{eV}$ energy loss per collision make a much smaller contribution. The $n_{e}$ gradient, which is less steep on the topside than on the bottomside, also adds a small amount to the spreading. However, overall Fig. 10 shows that Coulomb losses extend/move the location of the topside PL band to lower $n_{e}$, and as expected the lower the energy the greater the spreading to lower $n_{e}$ (e.g., Swartz et al. 1971).

The topside lines "a" and "b" in Figs. 8 and 9 have significant overlap similar to the corresponding 18.5 and $17.6 \mathrm{eV}$ peaks in Fig. 5; "b" corresponds to $17.6 \mathrm{eV}$, the smaller of the two peaks. The peaks at energies 18.5 and $17.6 \mathrm{eV}$ are also listed in Table 1 (column 1) along with their intensities (column 3). However, the separation between the peaks observed along the radar line-of-sight in Figs. 8 and 9 is nominally $0.7 \mathrm{eV}$ (i.e. $15.9 \mathrm{eV}$ measured for "a" minus $15.2 \mathrm{eV}$ measured for "b" in Fig. 10). With a small amount of broadening and energy offset the two peaks merge. For $n_{e}=5.5 \times 10^{-5}$ (Fig. 10) a TEC 


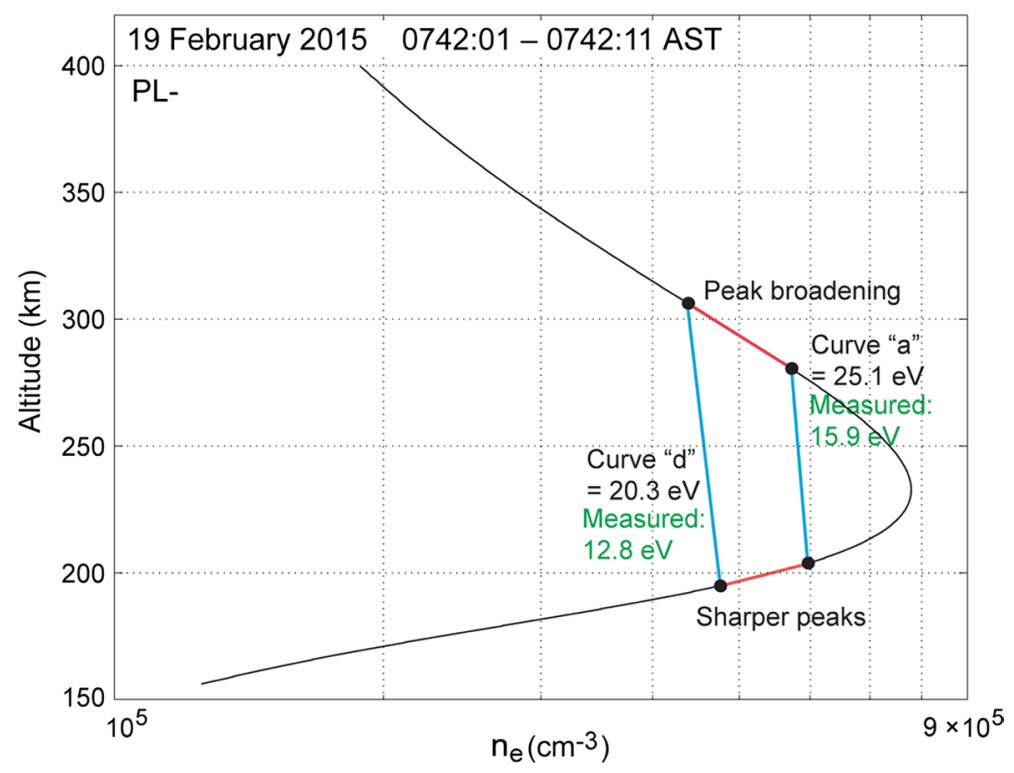

Fig. 10 Electron density $\left(n_{e}\right)$ profile obtained using the frequency of the spectral peak in the downshifted plasma line spectrum versus altitude. The technique used to obtain $n_{e}$ is the same as in Fig. 6 as are the designation of bottomside and topside spectral regions. The region of the $n_{e}$ profile where peaks and valleys occur as a result of solar EUV emissions is shown as a red overtrace on the bottomside $F$ region. A similar topside overtrace marks the broadened spectral structure. Curves "a" and "d" define the range of spectral peaks in Fig. 8 that are identified as $E_{\phi}$ at $\theta=0$. The $E_{\varnothing}$ values in green are the actual measured energy values of the spectral peaks at the beam zenith angle of $8.67^{\circ}$. For completeness, the actual measured values for curves " $c$ " and " $b$ " (not shown) are 14.0 and $15.2 \mathrm{eV}$, respectively. With the 10-s integration period used for the above $n_{e}$ calculation, the largest $1 \sigma n_{e}$ error at any $295-\mathrm{m}$ altitude cell in the plot is $0.024 \%$

path length of $\sim 50 \mathrm{~km}$ would be required to merge and broaden the two lines via Coulomb losses. As the $F$ region strengthens (Fig. 7) $n_{e} \propto f_{r}^{2}$ increases and the required path length decreases.

The North scan in Fig. 8 serves as a confirming test of $f_{r}(\theta)$ at large $\theta$. The results from the South zenith angle scan indicate that the PL strength decreases as $\theta$ increases, and thus the North signal should be very weak. In addition, Eq. (3) predicts that the signals will be either very high or very low in altitude where Langmuir wave losses are high. Nevertheless, weak signals were in fact observed on the North topside and are manifested as " $V$ " PL signatures in Fig. 8. The North lines correspond to three of the observed bottomside lines in the South (d, c, and b) as viewed at large $\theta=45.80^{\circ}-59.83^{\circ}$ angles. Two $V s$ marked "d" include the solid line prediction and the observed peak shown as a dotted line above the solid line. One $V$ reaches its nadir near 0845 AST and the other has a nadir near 0927 AST. In the North, signals near the nadir (i.e. near a zenith angle of $1.10^{\circ}$ ) have the lowest $\theta$ and therefore the greatest amplitude. Both are a branch of the observed $15.0 \mathrm{eV}\left(f_{r d}=6.58 \mathrm{MHz}, f_{r e}=6.62 \mathrm{MHz}\right)$ line discussed above in reference to the observed " $d$ " line in the South geomagnetic meridian scan, which has the corresponding $\theta=0$ value of $20.3 \mathrm{eV}$. Below the two curves labeled "d", the "c" curve and part of the "b" curve are discernable. Also note that 0845 AST a faint inverted "d" curve is seen very low in altitude (174 km altitude), which is also part 
of the $\theta=0,20.3 \mathrm{eV}$ branch seen in the South. This very weak curve has added losses because of its low altitude, which increases electron-neutral collisional losses compared to the corresponding topside line immediately above it. The topside signal at 0845 AST has the following parameters: $\theta=45.8^{\circ}, f_{r e}=6.05 \mathrm{MHz}$ at the nadir; and $\theta=59.8^{\circ}$, $f_{r e}=4.32 \mathrm{MHz}$ at the highest heights near the end of the detectable $V$. The weakness of the signal near nadir is primarily caused by Coulomb losses and the fact that the PE flux falls off rapidly as a function of $\theta$. The dotted line used to mark the observed PL- peak is located above the solid line predictions, which are calculated for no loss. The altitude displacement is indicative of moderate Coulomb losses. With increasing altitude along the $V$ the PE flux which generates the Langmuir waves is further reduced by Coulomb losses. In addition, electron thermal Landau damping of the PE-generated Langmuir waves in the $f_{r e}=4.32 \mathrm{MHz}-5.00 \mathrm{MHz}$ band is high (e.g., $Y P$ ), and this ultimately extinguishes the signal. The same arguments hold for the second $V$ signature near 0927 AST.

In Fig. 11, the PL- data of Fig. 8 is mapped to the format of $\kappa T_{p}$ versus $E_{\phi}$ and time. The squaring of $f_{r-}$ in arriving at $E_{\phi}$ provides increased sensitivity when comparing the measured PE spectral lines to those predicted by Eq. (3). In the South geomagnetic meridian plane, the overall agreement between the bottomside predictions shown as solid lines and labeled with the letters ( $a, b, c, d)$ and the observations is quite good. As discussed above the topside lines have an offset because of Coulomb losses. The weaker bottomside/topside structures observed at energies greater than a $=25.1 \mathrm{eV}$ correspond to the small peaks listed in column 5 of Table 1 as $27.5 \mathrm{eV}$ and $30.0 \mathrm{eV}$, and part of the

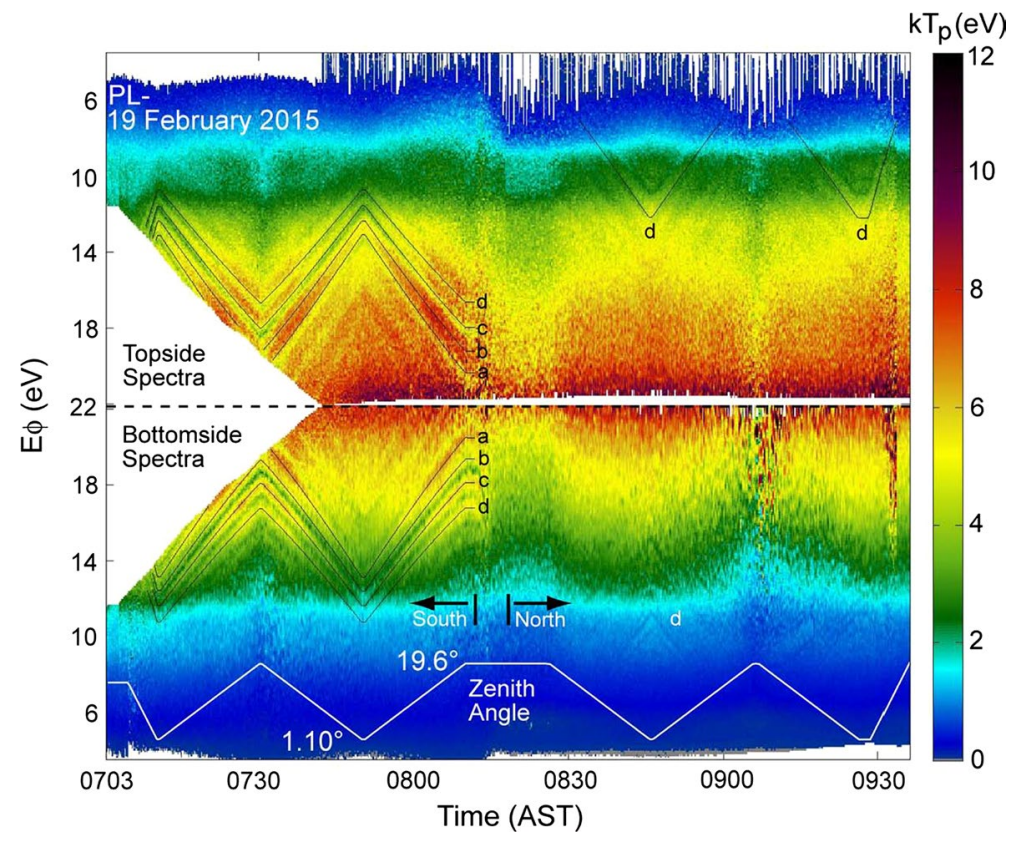

Fig. 11 The data of Fig. 8 presented in spectrogram form. The lower case letters have the same meaning as in Fig. 8. Lower case letters are used to identify the PE energy at $\theta=0$ that is responsible for the spectral line (Table 1, column 5). Thus, $a=25.1 \mathrm{eV}, b=23.9 \mathrm{eV}, c=22.1 \mathrm{eV}$, and $d=20.3 \mathrm{eV}$. See text for details 
broadened peak 31.8-35.2 eV. In the North geomagnetic meridian plane, topside "d", "c", and "b" lines are now evident, but only the "d" line is labeled for viewing purposes.

If Eq. (3) works for zenith scan data, it must also work on data acquired during azimuth scans. For validation, results from a "slow" azimuth scan performed at midday on 19 February 2015 are provided in Fig. 12. There is good agreement between the results of Eq. (3) plotted as solid lines and the data obtained in the bottomside $F$ region where Coulomb losses are very small. The $\theta$ values for these results range from $\sim 30.1^{\circ}$ at $0^{\circ}$ azimuth to $\sim 61.7^{\circ}$ at the two points labeled $\pm 180^{\circ} \mathbf{B}$ North. The usually weak $18.3 \mathrm{eV}$ line listed in Table 1 column 5, and designated as "e" in Fig. 12, is clearly evident in the observations. Most likely this is because of the decreased solar zenith angle $\left(\sim 30.5^{\circ}\right)$ during the noontime observations of Fig. 12 compared to the sunrise $\left(\sim 75^{\circ}\right)$ bottomside observations in Figs. 8, 9, and 11. In Fig. 12, all spectral lines are strongest at $0^{\circ}$ azimuth where the angle relative to $\mathbf{B}$ is smallest. At $0^{\circ}$, the "a" line is $200 \mathrm{~km}$ in altitude, and the "e" line is at a height of $183 \mathrm{~km}$. The well-defined $0^{\circ}$ spectral lines have greater $\kappa T_{p}$ values than the peaks measured at similar bottomside altitudes and $\theta$ in Fig. 11. The Fig. 12 spectral peaks are in the $\kappa T_{p}$ range of 6-12, whereas in Fig. 11 the range is 3-7. Thus, there is a factor of

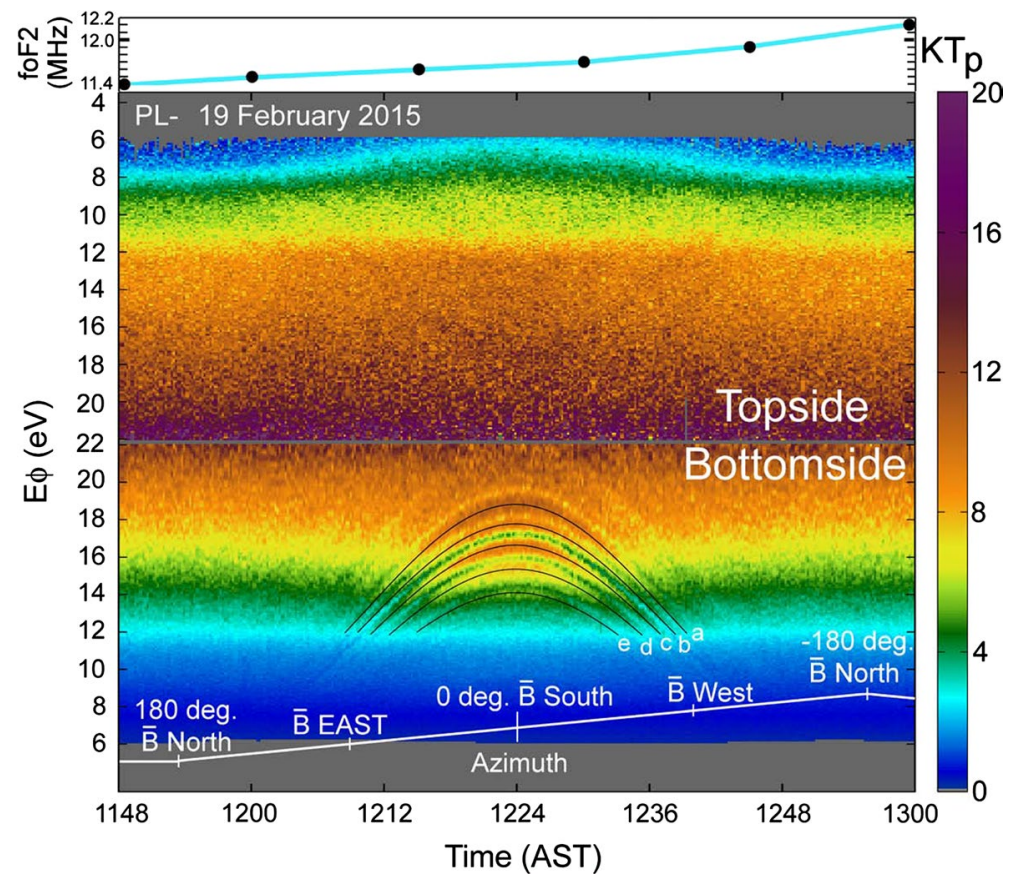

Fig. 12 Azimuth scan in spectrogram mode with the radar zenith angle fixed at $15^{\circ}$. The radar beam is slowly moved in azimuth at a rate of $5.76 \% \mathrm{~min}$ in a stable ionosphere. As in Fig. 8, the calculated EUVgenerated spectral peaks (black lines on bottomside) are identified as: $a=25.1 \mathrm{eV}, b=23.9 \mathrm{eV}, c=$ $22.1 \mathrm{eV}, d=20.3 \mathrm{eV}$, and in this case $e=18.3 \mathrm{eV}$. Except for " $a$ " these lines are neither broadened nor displaced because of low Coulomb losses. Curve " $a$ " is slightly broadened. Above " $a$ " a wide spectral structure is evident; this is the broadened bottomside peak at $31.8-35.2 \mathrm{eV}$ for $\theta=0$ in column 5 of Table 1 . The highly broadened spectrum on the topside is barely visible but is evident as a slightly darkened " $V$ " curve inverted relative to the bottomside observations. Although the topside curve meets our theoretical expectations, no calculated lines are shown because they distract from the view of the actual data. This is similar to the $V$ curves seen in Figs. 8, 9, and 11. Values for foF2 are plotted at the top of the display 
$\sim 2$ difference between $\kappa T_{p}$ values at sunrise and at noon. This ratio is consistent with the measured AE-E PE flux versus solar zenith angle listed in Table 1 of Lee et al. (1980a) for altitudes near $190 \mathrm{~km}$. Thus, the "e" curve is detected because of higher PE flux at noon.

The faint bottomside curves immediately above "a" in Fig. 12 are caused by two unresolved peaks at 27.5 and $30.0 \mathrm{eV}$ listed in column 5 of Table 1. The PL signals at and below "a" are detectable over $\pm 105^{\circ}$ in azimuth centered on the south meridian plane $\left(0^{\circ}\right)$. These signals disappear when the PL altitude falls below $\sim 150 \mathrm{~km}$ where the electronneutral collision frequency becomes large enough to fully damp the Langmuir waves. A topside inverted peak significantly broadened by Coulomb losses is barely discernable in the display. This peak corresponds to the small broadened topside peak at $\sim 18.5 \mathrm{eV}$ in Fig. 5 and agrees with the calculations of Eq. (3).

\subsection{Measurements of $\Delta f_{r}=\left(f_{r+}-f_{r-}\right)$}

It was discovered at the beginning of this project that when the radar beam was pointed South the $\Delta f_{r}=\left(f_{r+}-f_{r-}\right)$ profile at the bottomside peaks in $\kappa T_{p}\left(E_{\varnothing}\right)$ did not yield relatively smooth curves that are common to $\Delta f_{r}$ observations made with a vertically directed beam at AO (e.g., Nicolls et al. 2006). Figure 13 illustrates the averaging of raw difference data which yields altitude perturbations in the $\Delta f_{r}$ profile when the radar beam is pointed toward geomagnetic South at $31.6^{\circ}$ relative to B. As noted in Sect. 2.2 the spectral peaks and valleys observed in Fig. 5 have the special property of an $E_{\phi}$ dependence on pitch angle. Because $\Delta f_{r}$ contains information about the electron energy distribution function in the form of $T_{e}$, one might expect a new result in the difference profile at altitudes where the peaks and valleys are present. The South and North data segments in Fig. 13 were carefully selected so as to be very stable in time and spaced as closely as possible. Long $\sim 6 \mathrm{~min}$ segments were found on 9 February 2013 that met these criteria. Continuous and stable $\Delta f_{r}$ altitude perturbations from spectral peaks and valleys are evident in the raw data bracketed by horizontal lines in the South panel. As expected, these fluctuations are not evident in the North segment because the peak/valley spectral structures are extremely weak in this direction (e.g., Fig. 8). The time-averaged North and South $\Delta f_{r}$ data are displayed in the right panel of Fig. 13. This allows perturbations in the South (red overtrace) to be seen more clearly and referenced to the North profile. Other higher altitude structures in the red overtrace zone are weaker and are not readily seen in the raw data.

In Fig. 13 the North and South $\Delta f_{r}$ results in the right panel ignoring the overtrace are very similar below the $F$ region peak but diverge in the topside ionosphere. In both cases, the altitude of the $F$ region peak was $\sim 236 \mathrm{~km}$, and the peak frequency was $\sim 7 \mathrm{MHz}$. The spatial separation of the North and South topside ionospheres was $161 \mathrm{~km}$ at $300 \mathrm{~km}$ altitude. The solar zenith angle (SZA) for the South and North observations was $73.7^{\circ}$ and $71.0^{\circ}$, respectively. At these two SZAs there is a negligible difference in PE flux (e.g., Lee et al. 1980a). For instance, at $280 \mathrm{~km}$ altitude and above there is no change in PE flux for solar zenith angles greater than $80^{\circ}$. However, there was greater topside $n_{e}$ in the North versus the South by a factor of 1.15 , and therefore the electron temperature difference implied by $\Delta f_{r}$ is most likely caused by a difference in the electron cooling rate, which is proportional to $n_{e}^{2}$.

More detailed information is provided in Fig. 14. Here $\Delta f_{r}$ in $\mathrm{Hz}$ is expressed in terms of the linear measure of electron temperature (Showen 1979), that is, $T_{e}=\Delta f_{r} / 0.869 \sim 1.15$ $\Delta f_{r}$. This is denoted as $T_{e}\left(\Delta f_{r}\right)$. The bulk electron velocity along the radar line-of-sight is assumed to be negligible. In the three plots, black is used to indicate data obtained when the radar was pointed at a zenith angle of $15^{\circ}$ in the geomagnetic South meridian plane; 

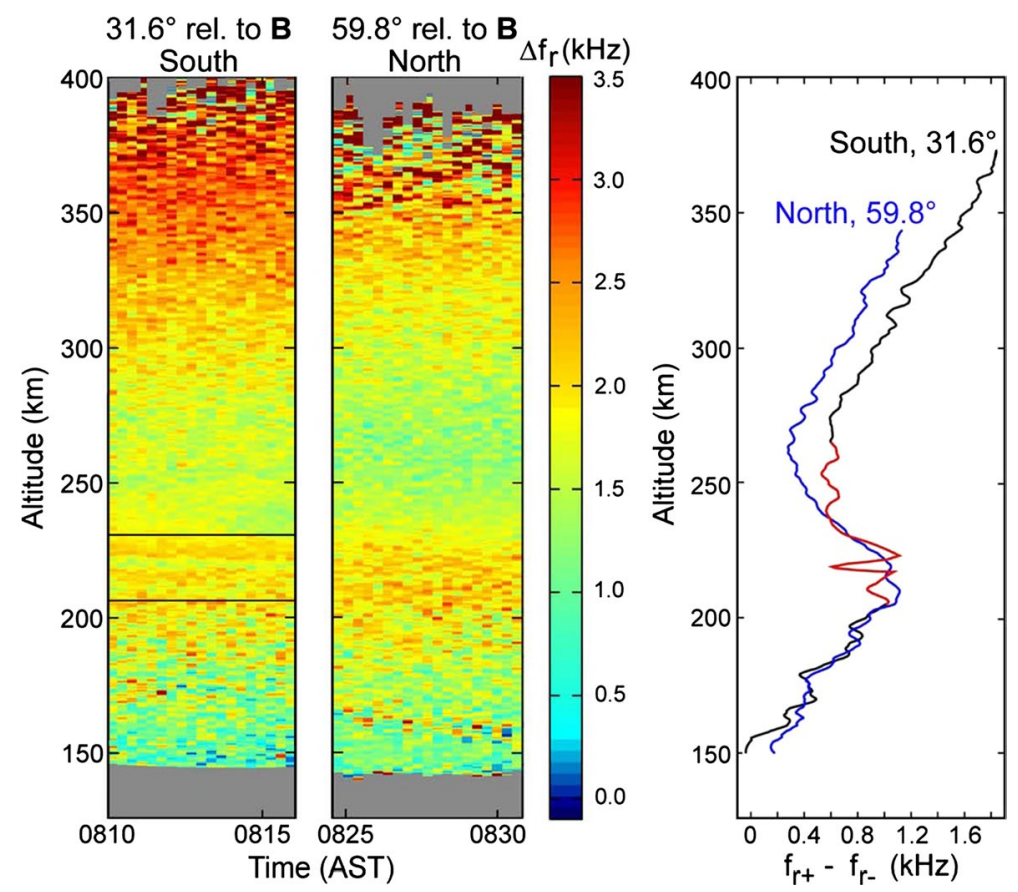

Fig. 13 Raw data showing $\Delta f_{r}$ versus altitude and time for $31.6^{\circ}$ and $59.8^{\circ}$ viewing angles relative to $\mathbf{B}$ (left panel). The South altitude region containing moderate $\Delta f_{r}$ fluctuations is demarcated by two horizontal lines. The gray color indicates areas where the reduced Chi squared fit probability was $<0.5 \%$ on either PL. Results within $10 \mathrm{~km}$ of the upper and lower edges of the displayed data have weaker signal strengths, and therefore have greater error bars than signals at other attitudes. Time integrated profiles of the data segments at $31.6^{\circ}$ and $59.8^{\circ}$ are presented in the panel on the right. The altitude region containing $\Delta f_{r}$ fluctuations is represented as a red overtrace in this panel. These data were acquired on 9 February 2013. Additional information concerning this observation is provided in the text and in Fig. 14c

the angle next to the word "South" is the angle of the radar beam relative to $\mathbf{B}$ (rounded to a tenth of a degree) at $\sim 225 \mathrm{~km}$ altitude, where the structure in the profiles resides. Black is also used for the ISR measured $T_{e}$ values, $T_{e}$ (ISR), the $T_{e}\left(\Delta f_{r}\right)$ profile, the time period of $T_{e}\left(\Delta f_{r}\right)$ data integration, the value of $f_{r}(\mathrm{MHz})$ at the $F$ region peak listed near the left altitude axis, and the altitude of the $F$ region peak, which is represented as a horizontal bar near the left axis. Blue refers to the above data obtained at a radar beam zenith angle of $15^{\circ}$ while looking North in the geomagnetic meridian plane. The small difference in the South/North beam angles relative to $\mathbf{B}$ in Panels A and B versus Panel C arises because of time varying changes in geomagnetic dip angle in the AO region. A 3D model based on the IGRF is used to determine B. The North and South data integration time periods were chosen to be as close as possible. The sparsely populated curves are $T_{e}$ (ISR) results from MRACF (38 km resolution) whereas the highly populated curves are the $T_{e}\left(\Delta f_{r}\right)$ data. Two subpanels in color on the bottom right of each panel show time segments of the PL+ and PL $-\kappa T_{p}\left(E_{\varnothing}\right)$ altitude profiles simultaneously acquired while looking South. These power profiles are similar to those in Fig. 8 except the current profiles are for constant zenith angle. The numbers on the left side of the PL- display identify the spectral peaks by observed electron energy in $\mathrm{eV}$ as indicated in column 1 of Table 1 . The mapping 
Fig. 14 Three panels used to illustrate the relationship between PEPL spectral lines/structure and the $f_{r+}$ minus $f_{r-}$ difference values expressed in terms of $T_{e}$. See text for details
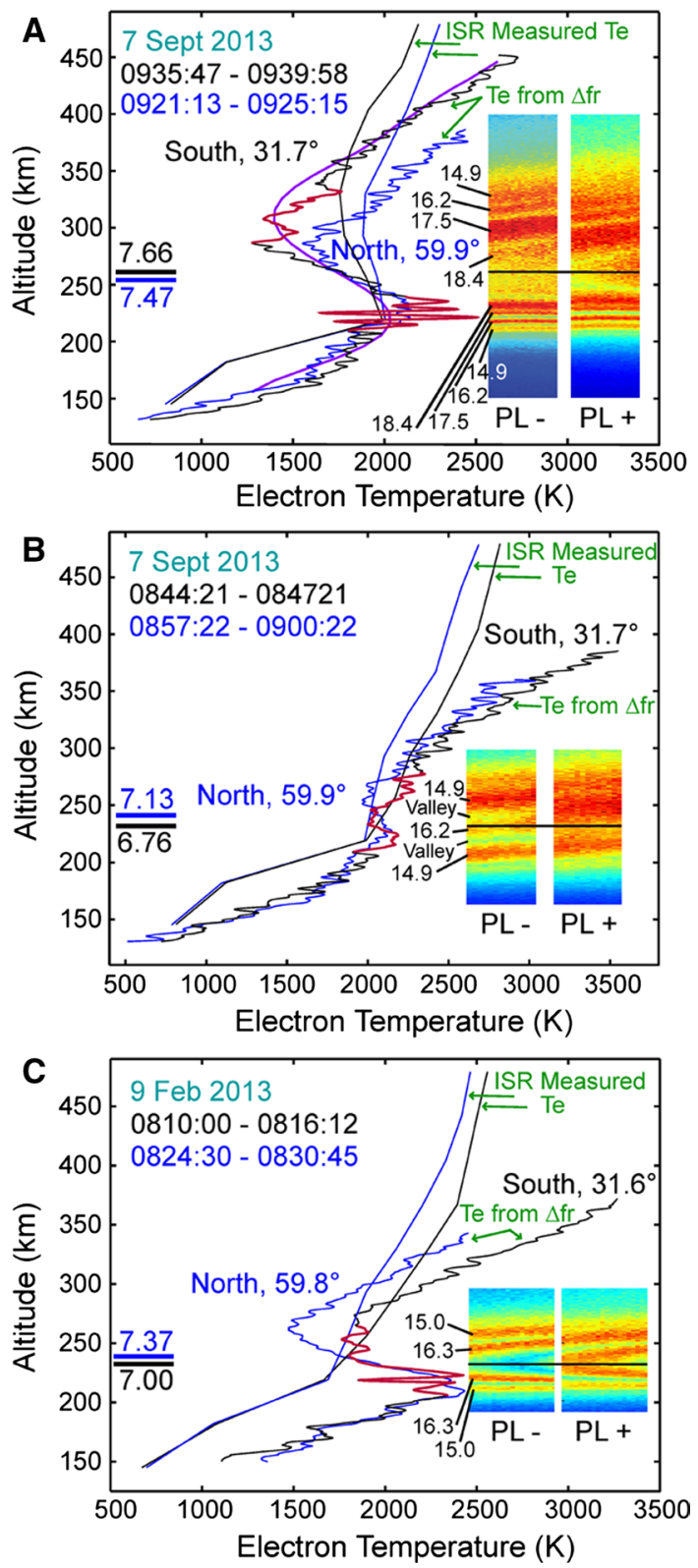

of the PL- energy values to the PL+ values is discussed below. There is a small difference of $-0.1 \mathrm{eV}$ between the column 1 energy values in Table 1 and the values listed in Panels $\mathrm{A}$ and $\mathrm{B}$. This is because the dip angle at the viewing location changed between 12 April 2012 (Table 1) and September 2013. All values shown are rounded to the nearest tenth of an $\mathrm{eV}$. The time interval of the PL profile data corresponds to the data integration times listed in the upper left corner of each panel, and the data are vertically aligned with the 
altitude axis. The horizontal black line across the subpanels represents the altitude of the $F$ region peak looking South. The subpanels are useful in determining how sharply the $\kappa T_{p}$ spectral peaks and valleys are defined in altitude and their relationship to $T_{e}\left(\Delta f_{r}\right)$. These spectral data are represented in a manner similar to that in Fig. 8. For the most part the vertical electron density gradient determines $\kappa T_{p}$ peak/valley separation distance on the altitude profile. However, as noted earlier Coulomb losses will broaden the peaks particularly on the topside $F$ region. As in Fig. 13, the red overtrace indicates the altitude interval(s) which contain spectral peaks and valleys.

In panel $\mathrm{A}$, the $T_{e}\left(\Delta f_{r}\right)$ data acquired at South $31.7^{\circ}$ reveals highly structured fluctuations (in red) that correspond to the $\kappa T_{p}$ peaks/valleys present in the altitude region between 211 and $230 \mathrm{~km}$. The maximum perturbations are $\pm 400 \mathrm{~K}$, which corresponds to $\pm 0.38 \mathrm{kHz}$ relative to the mean $\Delta f_{r}$ value of $2.4 \mathrm{kHz}(2083 \mathrm{~K})$. In general, on the bottomside the peak/valley $T_{e}\left(\Delta f_{r}\right)$ fluctuation is positive with respect to the mean when the $\mathrm{PL}+$ amplitude in the subplots is near a spectral peak, and the simultaneously measured $\mathrm{PL}-$ is near a spectral valley. The reverse is true for negative $T_{e}\left(\Delta f_{r}\right)$ perturbations. This entire sequence is reversed on the topside because the $n_{e}$ decreases with altitude. The above rule holds for all observations in Fig. 14. Note that there is always adequate signal in a spectral valley to determine the spectral frequency (e.g. blue curve in Fig. 5) and thereby obtain $T_{e}\left(\Delta f_{r}\right)$. In addition the greater the $n_{e}$ gradient, the narrower the $\kappa T_{p}$ peak is in altitude, and the resulting $\Delta f_{r}$ fluctuation is larger and sharper at altitudes where a PL+ peak/ valley is aligned with a PL- valley/peak. When shallow gradients are present $\Delta f_{r}$ fluctuations become weaker and broader in altitude. In cases where the peak/valley spectral peaks are not perfectly centered in the PL- and PL+ altitude cells a reduction of the enhanced $T_{e}\left(\Delta f_{r}\right)$ perturbations will occur. Also by virtue of Eq. (1) and the text immediately above and below it, we know that for constant Langmuir wave phase velocity, $v_{\phi}$, or equivalently for constant $E_{\phi}$, in the bottomside $F$ region the PL+ will always occur at an altitude greater than that of the PL- because $k_{+}>k_{-}$. The reverse is true for a topside gradient. This feature of the standard Langmuir wave dispersion relation also holds for the peaks/valleys discussed here. At higher altitudes in Panel A (red curve above $~ 286 \mathrm{~km}$ ) the fluctuations are smaller and broader mostly because of temporal variability of the peaks/valleys during the integration period. However, Coulomb losses and a shallow topside gradient also broaden the peaks/valleys, thereby reducing the amplitudes of the enhanced $T_{e}\left(\Delta f_{r}\right)$ perturbations.

Most likely the reason why there are enhanced $T_{e}\left(\Delta f_{r}\right)$ fluctuations is that the electron velocity distribution function is modified in the $\kappa T_{p}$ peaks and valleys resulting in perturbations in the $3 k^{2} \kappa T_{e} / m_{\mathrm{e}}$ term of the Langmuir wave dispersion relation. The background electron temperature measured with MRACF in the bottomside structured region in Panel A is $\sim 1989 \mathrm{~K}$, and $f_{r-}$ ranged from $\sim 6.8 \mathrm{MHz} \pm 0.5 \mathrm{MHz}$ in the region highlighted in red which corresponds to $E_{\phi}=\sim 14-19 \mathrm{eV}$. The $\pm 400 \mathrm{~K}$ spectral peak/valley perturbations, when compared to the background value of $\sim 1989 \mathrm{~K}$, indicates that the detected Langmuir waves were probably generated in a temperature environment far outside the normal electron-thermal velocity distribution function.

In this paragraph, we briefly discuss the standard application of $T_{e}\left(\Delta f_{r}\right)$ to determine $T_{e}$ in the $\mathrm{AO} F$ region. In panel $\mathrm{A}, T_{e}\left(\Delta f_{r}\right)$ agrees with the measured ISR $T_{e}$ values $T_{e}$ (ISR) only near the $F$ region peak plus one higher altitude location for both South $31.7^{\circ}$ and North $59.9^{\circ}$. In the following discussion we ignore the fluctuations in red caused by the $\kappa T_{p}$ peaks/valleys. A good match between the values $T_{e}\left(\Delta f_{r}\right)$ and $T_{e}$ (ISR) was not observed at Arecibo during the most recent solar maximum. This is evident in all panels in Fig. 14. The purple curve in panel A is calculated using Vlasov theory for a warm magnetized plasma for the South $T_{e}\left(\Delta f_{r}\right)$ measurements and represents a higher order correction to the $T_{e}\left(\Delta f_{r}\right)$ 
linear theory shown in the figure. The Vlasov solution requires numerical integration and an iterative approach to determine the relationship between $\Delta f_{r}$ and $T_{e}$. This approach is similar to that of the kinetic theory for a magnetized plasma (Farley et al. 1961) that is implemented by Nicolls et al. (2006). The Vlasov curve $T_{e}(\mathrm{~V})$ is a refinement of the linear calculations for $T_{e}\left(\Delta f_{r}\right)$, but neither curve matches the measured $T_{e}$ (ISR) data except near the $F$ region peak at $\sim 250 \mathrm{~km}$ and at $\sim 359 \mathrm{~km}$ altitude. $T_{e}\left(\Delta f_{r}\right)$ and $T_{e}(\mathrm{~V})$ implicitly assume that there is a magnetized electron thermal distribution function without PEs. It appears that additional refinements of the $T_{e}(\mathrm{~V})$ calculation are needed to explain the differences between $T_{e}(\mathrm{~V})$ and $T_{e}(\mathrm{ISR})$ in Fig. 14. The inclusion of a photoelectron tail to the thermal velocity distribution function (e.g., Guio et al. 1998) may provide the desirable match between theory and observations. The good agreement between $T_{e}\left(\Delta f_{r}\right)$, with $T_{e}$ calculated from kinetic theory, and $T_{e}$ (ISR) achieved near solar minimum by Nicolls et al. (2006) arises because of a significantly weaker ionosphere (lower $n_{e}$ at the $F$ region maximum) combined with a lower PE flux. This resulted in a dominant electron thermal plasma line for which the theoretical calculations were made, and hence the theory was in excellent agreement with the data at altitudes above $\sim 210 \mathrm{~km}$. The discrepancy between $T_{e}$ (ISR) and the calculated $T_{e}\left(\Delta f_{r}\right)$ from $\sim 150$ to $\sim 210 \mathrm{~km}$ is likely the result of the roles played by molecular ions, electron collisions, and photoelectrons in this altitude region.

Measurements made earlier in the day prior to those of panel A are presented in panel B. In this case only the first strong $\kappa T_{p} \mathrm{PL}-$ peak in column 1 of Table 1 at $E_{\phi}=15.0 \mathrm{eV}$ is fully visible and is located $\sim 25 \mathrm{~km}$ above and below the $F$ region peak (horizontal black line). As noted above, this peak is labeled $14.9 \mathrm{eV}$ in Fig. $14 \mathrm{~b}$ because the dip angle at the point of measurement changed between the time that the data of Table 1 were acquired (12 April 2012) and the date of the observations of Fig. 14b (3 September 2013). The change in dip angle changes $\theta$ in Eq. (3) and therefore $E_{\phi}$. The bottomside and topside $n_{e}$ gradients surrounding the $F$ region peak are shallow. The PL- valley between the 15.0 and $16.3 \mathrm{eV}$ peaks in Table 1 is evident immediately above the $14.9 \mathrm{eV}$ peak on the bottmside $F$ region and immediately below the $14.9 \mathrm{eV}$ peak on the topside. Exactly at the $F$ region peak, the PL- line panel shows that the second $\kappa T_{p}$ peak at $16.2 \mathrm{eV}$ is just beginning to emerge. The shallow gradients give rise to the "flattened" $T_{e}\left(\Delta f_{r}\right)$ perturbations shown in red. Note the reversal in $T_{e}\left(\Delta f_{r}\right)$ from maximum to minimum as one follows the red curve through the F region peak from below. With PL- valleys present immediately above and below the $F$ region peak, the $T_{e}\left(\Delta f_{r}\right)$ perturbation reverses from positive to negative relative to the mean in crossing the $F$ region peak. The topside broadening of PL- and PL+ signals at higher heights above $250 \mathrm{~km}$ is the result of Coulomb losses that generate overlap between peaks and valleys. This greatly decreases the $T_{e}\left(\Delta f_{r}\right)$ fluctuations shown in red.

A case with steeper bottomside and topside gradients near the $F$ region peak with the first two strong $\kappa T_{p}$ peaks $\left(E_{\phi}=15.0 \mathrm{eV}\right.$ and $\left.16.3 \mathrm{eV}\right)$ is presented in Panel C. This clearly shows that the PL+ occurs at a greater altitude than the PL- on the bottomside, and that the reverse is true for the topside. The decrease in amplitude of the $T_{e}\left(\Delta f_{r}\right)$ fluctuations shown in red on the topside is primarily the result of a strengthening topside ionosphere which gives rise to $\kappa T_{p}$ peaks and valleys that increase in altitude versus time during the integration period. This has the effect of reducing the $T_{e}\left(\Delta f_{r}\right)$ fluctuations.

\section{Discussion}

For many years there has been a mystery as to where the enhanced plasma line peaks from PEs generated by solar EUV line emissions were at AO. After all, the 
photoelectron peaks in the bottomside $F$ region and the topside peaks and structures smeared out by Coulomb losses were clearly evident in the AE-E data (e.g. Lee et al. $1980 \mathrm{a}, \mathrm{b}$ ) It is apparent from Fig. 8 that the plasma line peaks are very weak at a zenith angle of $1.10^{\circ}$. Thus, such peaks would not be noticeable when the radar beam was pointed near vertical. One would have to resolve the detection problem by dwelling and scanning the beam in the direction of geomagnetic South. In the current case, our motivation for looking both South and North for prolonged periods of time was to perform a high-resolution electron thermal balance study (e.g., Carlson et al. 1977) in the upper $F$ region. This led to the unanticipated discoveries presented in this paper.

We have demonstrated that the solar EUV line generated PL peaks and structures obtained from radar observations of Langmuir waves have a dependence on $\theta$, the pitch angle, where $\mathbf{B}$ is determined from the IGRF model. It is important to address the accuracy of the dip angle in this model, and whether the dip angle function discussed in Sect. 2.2 should be $f_{r}(\theta)=A \cos (\theta)^{1.0}$ and $E_{\phi}(\theta)=D \cos (\theta)^{2.0}$ instead of the derived $f_{r}(\theta)=A \cos (\theta)^{0.97}$ and $E_{\varnothing}(\theta)=D \cos (\theta)^{1.94}$. The latest version of the IGRF model field (12th generation) was used for the current calculations, and a 3D model of the geomagnetic field was developed from the IGRF. The dip angles used in the above calculations would have to increase by $1^{\circ}$ for the exponents to be changed from 0.97 and 1.94 to 1.0 and 2.0, respectively. This would imply that there is an unrealistically large error in the IGRF. According to the IGRF, the secular variation in the dip angle immediately above $\mathrm{AO}$ is $0.185^{\circ}$ year. Temporal changes of the geomagnetic field are taken into account at all locations sampled by the AO radar. The 2012, 2013, and 2015 data presented here were obtained under geomagnetic quiet conditions of $\mathrm{Kp}=2-$ or less, and the solar sunspot numbers were 37 (April 2012), 38 (Feb. 2013), 25 (Sept. 2013), and 61 in (Feb. 2015), so any magnetic field fluctuations external to the IGRF model were very small. The estimated IGRF dip angle error based on the on-line IGRF "health warning" during both observations is no greater than $\pm 0.05^{\circ}$, which has negligible impact on the results. The error in not including 3D lateral changes in the dip angle and using the time varying dip angle profile above $\mathrm{AO}$ instead results in exponents that are too low by 0.02 and 0.04 , that is, $\cos (\theta)$ exponents of 0.95 and 1.90 , which do not adequately fit the data. Overall, the experimentally derived PL dip angle function is satisfied for the measured range of $\theta$ values from $27.6^{\circ}$ to $64.6^{\circ}$. Moreover, the projected $E_{\phi}(\theta=0)$ appears to be correct because the $E_{\phi}$ of the peaks in Fig. 5 are well-matched to the energy locations of the AE-E PE peaks (Table 1 columns 5,6). Most likely the dip angle function is satisfied over a wider range of $\theta$ than that measured here.

The AE-E satellite results clearly indicate that the peaks in the photoelectron fluxes are generated on a background photoelectron flux spectrum that decreases exponentially with increasing energy due to an energy cascade (e.g., Lee et al. 1980a, b; Hernandez et al. 1983). Also, "for the near-equatorial orbit of AE-E, the sensor look direction is approximately parallel to the magnetic field direction in most cases" (Doering et al. 1976). It is possible to obtain a PE spectrum from the $\kappa T_{p}$ spectrum in Fig. 5 by adding boundary conditions to Eq. (2) and solving for $f_{\mathrm{p}}\left(E_{\phi}\right)$. This generates a one-dimensional PE velocity distribution function, from which a $3-\mathrm{D}$ distribution function can be deduced. However, as indicated in Sect. 2.1, Eq. (2) must first be corrected to account for PEs at energies $>30 \mathrm{eV}$. Also the current $\kappa T_{p}$ spectral data are highly structured and are not amenable to the $Y P$ inversion technique (i.e., $Y P$ Eq. (18)) for converting $\kappa T_{p}$ spectra into $1-\mathrm{D} P E$ velocity distribution functions. Other approaches such as the modeling implemented by Guio et al. (1998) and Bjørnå and Trulsen (1986) may be more appropriate for future work in $\kappa T_{p}$ to PE flux conversion. 
The fact that the bottomside PE spectral peaks reside on the background PE electron energy distribution function that decreases with energy (Lee et al. 1980a,b) may mean that the PL peaks generated by the PEs are unstable with respect to the bump-on-tail instability (e.g., Cap 1978; Dum 1989; Chen 1990; Bittencourt 2004). Langmuir waves with $E_{\varnothing}$ on a positive slope in the PE distribution function $\left(\partial f_{p e} / \partial E_{\phi}\right)$ will be unstable, and energy will be transferred from the particles to the wave via electron Landau damping. Positive slopes in the PE distribution function near EUV peaks are evident in the AE-E database (e.g., Lee et al. 1980a, b). The Langmuir wave spectral peaks shown for example in Fig. 5 owe their existence to solar EUV line emissions, but we note that they are located in a prime environment for the development of the kinetic bump-on-tail instability. In addition, the spectral peaks may be viewed as electron beams of moderate amplitudes propagating in the background PE energy distribution. In this case the geomagnetic field and electron density gradients complicate electron beam propagation in the ionosphere, and it is feasible for two different processes/instabilities, one parallel and one perpendicular to $\mathbf{B}$, to give rise to a competition between beam-induced diffusion processes (e.g., Ghizzo et al. 1993). Although the Ghizzo et al. study is not directly applicable to the current PE observations because of differences in scaled plasma parameters, it demonstrates the complexity of the beam-plasma approach. Whether this or other diffusion processes can alter the $E_{\phi}$ pitch angle distribution at the Langmuir wave peaks and valleys and explain the current results is a question to be resolved in future experiments and simulations. In addition, based on the locations of the electron cyclotron harmonics in Fig. 5, we know that electron cyclotron instabilities do not play a role in the measurements. Other unspecified instabilities may take place in the plasma. However, any suggested instability process must explain why the $E_{\phi}(\theta)$ pitch angle distribution of Langmuir waves in the spectral peaks/valleys is not isotropic but takes on the form $E_{\phi}(\theta)=D \cos (\theta)^{1.94}$. At present, no known simulation or theory exists that can be directly applied to the PL observations presented here.

In Sect. 2.1 it was noted that the $\kappa T_{p}\left(E_{\varnothing}\right)$ in Fig. 5 has a substantial value at $E_{\varnothing}=51 \mathrm{eV}$ $\left(\kappa T_{p}=7.1 \mathrm{eV}\right)$, which is well above the prediction of $Y P$. The origin of this discrepancy can be traced back to the theory of Perkins and Salpeter (1965), who in their Eq. (46) assume that the high energy portion of the PE tail $\left(E_{\varnothing}>30 \mathrm{eV}\right)$ can be replaced by a Maxwellian. The Maxwellian generates thermal Langmuir waves that have amplitudes much less than those of PEs. At least in part, this was done because of a document cited in a footnote of Perkins and Salpeter (1965) as: Mariani, F., Preliminary Report, Goddard Space Flight Center, Greenbelt, Maryland (unpublished). The report indicated that the PE flux fell off steeply at PE energies above $30 \mathrm{eV}$. If the formalism of $Y P$ were correct one would have the thermal level $\kappa T_{p}\left(E_{\phi}\right) \sim \kappa T_{e}=0.013 \mathrm{eV}$ at $E_{\phi}=51 \mathrm{eV}$, which is much less than the observed $\kappa T_{p}$ of $7.1 \mathrm{eV}$. In addition, the question arises for the measured $7.1 \mathrm{eV}$ value as to whether PEs are totally responsible for this excitation level, or whether plasma damping processes play a role in lowering this value below the nominal PE level. According to Perkins and Salpeter (1965) the PL will be "fully" enhanced if the electron Landau damping generated by the PEs (which increases the amplitude of the Langmuir wave) greatly exceeds losses resulting from electron Landau damping from the background PE distribution function. Perkins and Salpeter (1965) calculate the ratio between PL enhancement and PL damping in their Eq. (50) to be $~ 30 \gg 1$ for $E_{\varnothing}<30 \mathrm{eV}$, and therefore full enhancement is achieved. For greater energies near $E_{\phi}=51 \mathrm{eV}$ one must include losses from electron-ion collisions $\nu_{e i}$ in the denominator of Eq. (50). Near $E_{\phi}=51 \mathrm{eV}$ the $\nu_{e i}$ losses obtained from Fig. 2 dominate the electron Landau damping losses produced by the background PE distribution. With the measured values of $n_{e}=2 \times 10^{6}$ and $T_{e}=1200 \mathrm{~K}$ at the excitation altitude $(314 \mathrm{~km})$, one obtains $\nu_{e i} \sim 2700 \mathrm{~s}^{-1}$. This combined with our 
estimate of $\chi_{p h}\left(E_{\phi}=51 \mathrm{eV}\right)=\sim 10^{-8}$ in Eq. (50) yields a ratio of $\sim 6$, which indicates that the full PL enhancement may have been achieved. In the future, an estimate of the PE energy spectrum at energies $>30 \mathrm{eV}$ obtained using $\kappa T_{p}$ versus $E_{\phi}$ data and a revised Eq. (2) will be employed to better assess PL losses via $\nu_{e i}$.

\section{Conclusions}

For the first time, the full photoelectron-enhanced plasma line spectrum (i.e. $\kappa T_{\mathrm{p}}$ versus electron phase energy $E_{\varnothing}$ ) has been captured at Arecibo with the aid of the Gregorian feed system. The high $F$ region electron densities (foF2 $\sim 12.4 \mathrm{MHz}$ ) present at solar maximum near the vernal equinox supported the determination of $\kappa T_{\mathrm{p}}$ out to $E_{\varnothing}=51 \mathrm{eV}$. The plasma line spectrum is highly structured with peaks and valleys having energies between $\sim 14$ and $\sim 27 \mathrm{eV}$ at altitudes below $\sim 245 \mathrm{~km}$. The spectrum contains information about the generation of Langmuir waves in response to photoelectrons produced by Mg IX and He II solar line emissions. The topside spectra can be broadened/smeared out between $E_{\emptyset} \sim 14$ and $\sim 27 \mathrm{eV}$ as a result of Coulomb PE-thermal electron energy loss. The locations of the bottomside peaks when scaled to the parallel-to-B geometry agree with those observed with the PE spectrometer on AE-E. A major result is that PL peaks and valleys measured with the radar follow the pitch angle function $E_{\phi}(\theta)=D \cos (\theta)^{1.94}$, or equivalently $f_{r}(\theta)=A \cos (\theta)^{0.97}$, where $\theta$ is pitch angle and $D$ and $A$ are normalization constants. These formulae are very sensitive to the geomagnetic dip angle, and an accurate 3-D field model is required for the calculation. The above formulae are valid for broadened topside PL structures after corrections are made for Coulomb PE-thermal electron energy losses. New theoretical approaches that include a magnetized plasma are needed to explain these results. One possible route is to extend the formalism of Guio et al. (1998) to directions oblique to the magnetic field. Simulations/theoretical efforts aimed at determining how a bump-on-tail instability develops in the ionosphere in the presence of the multi-peaked PE distribution function are highly desirable. In addition, the potential competition between PE "beam-induced" diffusion parallel and perpendicular to $\mathbf{B}$ should be addressed as part of a future theoretical study. Additional insight into the above problem is provided by the Langmuir wave frequency difference $\Delta f_{r}=\left(f_{r+}-f_{r-}\right)$, which exhibits large fluctuations in the altitude region of peaks and valleys. Most likely the electron temperature distribution function is modified in this zone resulting in a perturbation of the Langmuir wave dispersion relation.

The measured continuum PL spectrum above $~ 30 \mathrm{eV}$ highlights a discrepancy in the theory of Perkins and Salpeter (1965) in which the PE production spectrum was assumed to fall off rapidly after $\sim 30 \mathrm{eV}$, and therefore the PE spectral tail was replaced by a Maxwellian. The sharp fall off does not exist (e.g., Lee et al. 1980a, b), and the current data clearly shows that $\kappa T_{\mathrm{p}}$ values near $E_{\varnothing}=51 \mathrm{eV}$ are a factor of 69 greater than the theorical prediction of Perkins and Salpeter. Thus, Eq. (7) of Yngvesson and Perkins (1968) must be corrected prior to future use at $E_{\varnothing}$ values greater than $30 \mathrm{eV}$. We also note that a better methodology than the simple inversion in Eq. (18) of Yngvesson and Perkins (1968) is needed to convert $\kappa T_{\mathrm{p}}$ versus $E_{\varnothing}$ into a one-dimensional photoelectron velocity distribution function with the aid of experimentally guided initial values for PE fluxes. Currently, a very smooth $\kappa T_{\mathrm{p}}$ versus $E_{\varnothing}$ curve is required, which is inconsistent with the present observations. Overall, the daytime PL observations reported here have led to several unexpected theoretical challenges that will be addressed as part of future collaborative studies. 
Acknowledgements The data in this paper is made available to the research community by Arecibo Observatory beginning 18 months after the date that the data is acquired. FTD and LDZ acknowledge support from the NSF under Grant AGS-1012006, and HCC acknowledges support under NSF Grant AGS1011921. The engineering and computer support of Michael P. Sulzer, Nester Aponte, and Phil Perillat is gratefully acknowledged. The Arecibo Observatory is operated by SRI International under a cooperative agreement with the National Science Foundation (AST-1100968), and in alliance with Ana G. MéndezUniversidad Metropolitana, and the Universities Space Research Association.

Open Access This article is distributed under the terms of the Creative Commons Attribution 4.0 International License (http://creativecommons.org/licenses/by/4.0/), which permits unrestricted use, distribution, and reproduction in any medium, provided you give appropriate credit to the original author(s) and the source, provide a link to the Creative Commons license, and indicate if changes were made.

\section{References}

V.J. Abreu, H.C. Carlson, Photoelectron energy loss and spectral features deduced by the plasma line technique. J. Geophys. Res. 82, 1017-1023 (1977)

N. Aponte, M.P. Sulzer, M. Nicolls, R. Nikoukar, S.A. Gonzalez, Molecular ion composition measurements in the F1 region at Arecibo. J. Geophys. Res. 112, A06322 (2007). https://doi.org/10.1029/2006J A012028

P. Bauer, K.D. Cole, G. Lejeune, Field aligned electric currents and their measurement by the incoherent scatter technique. Planet. Space Sci. 24, 479-485 (1976)

R.A. Behnke, S. Ganguly, First direct ground-based measurement of electron drift in the ionospheric $F$ region. J. Geophys. Res. 91, 10178-10182 (1986)

A.N. Bhatt, M.J. Nicolls, M.P. Sulzer, M.C. Kelley, Observations of plasma line splitting in the ionospheric incoherent scatter spectrum. Phys. Rev. Lett. (2008). https://doi.org/10.1103/PhysRevLett.100.045005

J.A. Bittencourt, Fundamentals of Plasma Physics, 3rd edn. (Springer, New York, 2004)

N. Bjørnå, Derivation of ion-neutral collision frequencies from a combined ion line/plasma line incoherent scatter experiment. J. Geophys. Res. 94, 3799-3804 (1989)

N. Bjørnå, S. Kirkwood, Observations of natural plasma lines in the E-region and lower F-region with the EISCAT UHF radar. Ann. Geoeophys. 4, 137-144 (1986)

N. Bjørnå, S. Kirkwood, Derivation of ion composition from a combined ion line/plasma line incoherent scatter experiment. J. Geophys. Res. 93, 5787-5793 (1988)

N. Bjørnå, J. Trulsen, Effect of a power-law particle flux on the ionospheric incoherent scattering cross section. Phys. Scr. 33, 284-288 (1986)

F. Cap, Handbook on Plasma Instabilities, vol. 2 (Academic Press, New York, 1978)

H.C. Carlson, V.B. Wickwar, G.P. Mantas, The plasma line revisited as an aeronomical diagnostic: suprathermal electrons, solar EUV, electron-gas thermal balance. Geophys. Res. Lett. 4, 565-567 (1977)

F.F. Chen, Introduction to Plasma Physics and Controlled Fusion, 2nd edn. (Plenum Press, New York, 1990)

R.J. Cicerone, Photoelectrons in the ionosphere: radar measurements and theoretical computations. Rev. Geophys. 12, 259-271 (1974)

R.J. Cicerone, W.E. Swartz, R.S. Stolarski, A.F. Nagy, J.S. Nisbet, Thermalization and transport of photoelectrons: a comparison of theoretical approaches. J. Geophys. Res. 78, 6709-6725 (1973)

F.T. Djuth, M.P. Sulzer, J.H. Elder, Application of the coded long pulse technique to plasma line studies of the ionosphere. Geophys. Res. Lett. 21, 2725-2728 (1994)

F.T. Djuth, M.P. Sulzer, J.H. Elder, V.B. Wickwar, High-resolution studies of atmosphere-ionosphere coupling at Arecibo Observatory, Puerto Rico. Radio Sci. 32, 2321-2344 (1997)

F.T. Djuth, M.P. Sulzer, S.A. Gonzales, J.D. Mathews, J.H. Elder, R.L. Walterscheid, A continuum of gravity waves in the Arecibo thermosphere? Geophys. Res. Lett. 31, L16801 (2004). https://doi. org/10.1029/2003GL019376

F.T. Djuth, L.D. Zhang, D.J. Livneh, I. Seker, S.M. Smith, M.P. Sulzer, J.D. Mathews, R.L. Walterscheid, Arecibo's thermospheric gravity waves and the case for an ocean source. J. Geophys. Res. 115, A08305 (2010). https://doi.org/10.1029/2009JA014799

J.P. Doering, C.O. Bostrom, J.C. Armstrong, The photoelectron-spectrometer experiment on Atmosphere Explorer. Radio Sci. 8, 387-392 (1973)

J.P. Doering, W.K. Peterson, C.O. Bostrom, T.A. Potemra, High resolution photoelectron energy spectra from AE-E. Geophys. Res. Lett. 3, 129-131 (1976) 
C.T. Dum, Transition in the dispersive properties of beam-plasma and two-stream instabilities. J. Geosphys. Res. 94, 2429-2442 (1989)

J.W. Evans, Theory and practice of ionosphere study by Thomson scatter radar. Proc. IEEE 57, 496 (1969)

D.T. Farley, J.P. Dougherty, D.W. Barron, A theory of incoherent scattering of radio waves by a plasma: II. Scattering in a magnetic field. Proc. R. Soc. Ser. A. 263, 238-258 (1961)

$\AA$. Fredriksen, High latitude quiet summer ion composition profiles derived from a combined ion line/ plasma line incoherent scatter experiment. J. Atmos. Terr. Phys. 52, 77-84 (1990)

Å. Fredriksen, C. La Hoz, Measurements of chirped natural plasma lines with EISCAT. Adv. Space Sci. 12(6), 231-234 (1992). https://doi.org/10.1016/0273-1177(92)90062-3

Å. Fredriksen, N. Bjørnå, T.L. Hansen, First EISCAT two radar plasma line experiment. J. Geophys. Res. 94, 2727-2731 (1989)

Å. Fredriksen, N. Bjørnå, J. Lilensten, Incoherent scatter plasma lines at angles with the magenetic field. J. Geophys. Res. 97, 16921-16933 (1992)

E.J. Fremouw, J. Petriceks, F.W. Perkins, Thomson scatter measurements and magnetic field effects on the Landau damping and excitation of plasma waves. Phys. Fluids 12, 869-874 (1969)

A. Ghizzo, P. Bertrand, M. Shoucri, E. Fijalkow, M. Feix, Study of the diffusion across a magnetic field in a beam-plasma interaction using a drift-kinetic Vlasov code. Phys. Fluids B 5, 4312-4326 (1993)

L. Goembel, J.P. Doering, D. Morrison, L.J. Paxton, Atmospheric O/N2 ratios from photoelectron spectra. J. Geosphys. Res. 102, 7411-7419 (1997)

P. Guio, W. Kofman, Alternating-code experiment for plasma-line studies. Ann. Geophys. 14, 14731479 (1996)

P. Guio, J. Lilensten, Effect of suprathermal electrons on the intensity and Doppler frequency of electron plasma lines. Ann. Geophys. 17, 903-912 (1999)

P. Guio, J. Lilensten, W. Kofman, N. Bjorna, Electron velocity distribution function in a plasma with temperature gradient and in the presence of suprathermal electrons: application to incoherent-scatter plasma lines. Ann. Geophys. 16, 1226-1240 (1998)

T. Hagfors, M. Lehtinen, Electron temperature derived from incoherent scatter radar observation of the plasma line frequency. J. Geophys. Res. 86, 119-124 (1981)

T. Hagfors, W. Birkmayer, M. Sulzer, A new method for accurate ionospheric electron density measurements by incoherent scatter radar. J. Geophys. Res. 89, 6841-6845 (1984)

C.J. Heinselman, J.F. Vickrey, On the frequency of Langmuir waves in the ionosphere. J. Geophys. Res. 97, 14905-14910 (1992a)

C.J. Heinselman, J.F. Vickrey, On the spectral analysis and interpretation of incoherent scatter plasma line echoes. Radio Sci. 27, 221-230 (1992b)

S.P. Hernandez, J.P. Doering, V.J. Abreu, G.A. Victor, Comparison of absolute photoelectron fluxes measured on AE-C and AE-E with theoretical fluxes and predicted and measured N2 2PG $3371 \AA$ volume emission rates. Planet. Space Sci. 31, 221-233 (1983)

S. Kirkwood, N. Bjørnå, Electron temperatures determined by tristatic plasma-line observations with the EISCAT UHF incoherent scatter radar. Geophys. Res. Lett. 19, 661-664 (1992)

W. Kofman, G. Lejeune, Determination of low-energy photoelectron distribution from plasma line measurements at Saint Santin. Planet. Space Sci. 28, 661-673 (1980)

W. Kofman, G. Lejeune, T. Hagfors, P. Bauer, Electron temperature measurements by the plasma line technique at the French incoherent scatter radar facilities. J. Geophys. Res. 86, 6795-6801 (1981)

W. Kofman, J.-P. St-Maurice, A.P. van Eyken, Heat flow effect on the plasma line frequency. J. Geophys. Res. 98, 6079-6085 (1993)

J.S. Lee, J.P. Doering, C.O. Bostrom, T.A. Potemra, Measurement of the daytime photoelectron energy distribution from AE-E with improved energy resolution. Geophys. Res. Lett. 5, 581-583 (1978)

J.S. Lee, J.P. Doering, T.A. Potemra, L.H. Brace, Measurements of the ambient photoelectron spectrum from Atmosphere Explorer, I, AE-E measurements below $300 \mathrm{~km}$ during solar minimum conditions. Planet. Space Sci. 28, 947-971 (1980a)

J.S. Lee, J.P. Doering, T.A. Potemra, L.H. Brace, Measurements of the ambient photoelectron spectrum from Atmosphere Explorer, II, AE-E measurements from 300 to $1000 \mathrm{~km}$ during solar minimum conditions. Planet. Space Sci. 28, 973-996 (1980b)

J. Lei, R.G. Roble, W. Wang, B.A. Emery, S.-R. Zhang, Electron temperature climatology at Millstone Hill and Arecibo. J. Geophys. Res. 112, A02302 (2007). https://doi.org/10.1029/2006JA012041

J. Lilensten, J.W. Kofman, I. Wisenberg, E.S. Oran, C.R. Devore, Ionization efficiency due to primary and secondary photoelectrons: a numerical model. Ann. Geophys. 7, 83-90 (1989)

D.J. Livneh, I. Seker, F.T. Djuth, J.D. Mathews, Continuous quasiperiodic thermospheric waves over Arecibo. J. Geophys. Res. 112(A7), A07313 (2007). https://doi.org/10.1029/2006JA012225 
D.J. Livneh, I. Seker, F.T. Djuth, J.D. Mathews, Omnipresent vertically coherent fluctuations in the ionosphere with a possible worldwide-midlatitude extent. J. Geophys. Res. 114(A6), A06303 (2009). https ://doi.org/10.1029/2008JA013999

G.P. Mantas, H.C. Carlson, V.B. Wickwar, Photoelectron flux build-up in the plasmasphere. J. Geophys. Res. 83, 1-15 (1978)

E. Mishin, T. Hagfors, On heat flow contribution to plasma line frequency in the F region. J. Geophys. Res. 99, 6537-6539 (1994)

A.L. Newman, E.S. Oran, The effects of electron-neutral collisions on the intensity of plasma lines. J. Geophys. Res. 86, 4790-4794 (1981)

M.J. Nicolls, M.P. Sulzer, N. Aponte, R. Seal, R. Nikoukar, S.A. Gonzalez, High-resolution electron temperature measurements using plasma line asymmetry. Geophys. Res. Lett. 33, L18107 (2006). https:// doi.org/10.1029/2006GL027222

M.J. Nicolls, S.L. Vadas, N. Aponte, M.P. Sulzer, Horizontal parameters of daytime thermospheric gravity waves and $E$ region neutral winds over Puerto Rico. J. Geophys. Res. Space Phys. 119, 575-600 (2014). https://doi.org/10.1002/2013JA018988

E.S. Oran, P.J. Palmadesso, S. Ganguly, Low-altitude plasma line anisotropy. J. Geophys. Res. 83, 21902194 (1978)

E.S. Oran, V.B. Wickwar, W. Kofman, A. Newman, Auroral plasma lines: a first comparison of theory and experiment. J. Geophys. Res. 86, 199-205 (1981)

F.W. Perkins, E.E. Salpeter, Enhancement of plasma density fluctuations by non-thermal electrons. Phys. Rev. 139, A55-A62 (1965)

E.E. Salpeter, Plasma density fluctuations in a magnetic field. Phys. Rev. 122(6), 1663-1674 (1961)

A. Savitzky, J.E. Golay, Smoothing and differentiation of data by simplified least squares procedures. Anal. Chem. 36(8), 1627-1639 (1964). https://doi.org/10.1021/ac60214a047

R.W. Schunk, P.B. Hays, Photoelectron energy loss to thermal electrons. Planet. Space Sci. 19, 113-117 (1971)

R.W. Schunk, A.F. Nagy, Ionospheres: Physics, Plasma Physics, and Chemistry, Cambridge Atmospheric and Space Science Series, 2nd edn. (Cambridge University Press, Cambridge, 2009)

R.L. Showen, The spectral measurement of plasma lines. Radio Sci. 14, 503-508 (1979)

M.P. Sulzer, A radar technique for high range resolution incoherent scatter autocorrelation function measurements utilizing the full power of klystron radars. Radio Sci. 21, 1033-1040 (1986a)

M.P. Sulzer, A phase modulation technique for a sevenfold statistical improvement in incoherent scatter data-taking. Radio Sci. 21, 737-744 (1986b)

W.E. Swartz, J.S. Nishbet, A.E.S. Green, Analytic expression for the energy-transfer rate from photoelectrons to thermal-electrons. J. Geophys. Res. 76, 8425-8426 (1971)

D. Vidal-Madjar, Theoretical study of propagation of short periodic gravity waves within the thermosphere: application to the plasma line measurement and to a Faraday rotation experiment. J. Atmos. Terr. Phys. 40, 1-12 (1978)

V.B. Wickwar, The $6300 \AA$ predawn enhancement: excitation by photoelectrons from the magnetic conjugate point. Ann. Geophys. 27, 187-192 (1972)

K.O. Yngvesson, F.W. Perkins, Radar Thomson scatter studies of photoelectrons in the ionosphere and Landau damping. J. Geophys. Res. 73, 97-110 (1968) 